\title{
INVERSE SCATTERING FOR THE LAPLACE OPERATOR WITH BOUNDARY CONDITIONS ON LIPSCHITZ SURFACES
}

\author{
ANDREA MANTILE AND ANDREA POSILICANO
}

\begin{abstract}
We provide a general scheme, in the combined frameworks of Mathematical Scattering Theory and Factorization Method, for inverse scattering for the couple of selfadjoint operators $(\widetilde{\Delta}, \Delta)$, where $\Delta$ is the free Laplacian in $L^{2}\left(\mathbb{R}^{3}\right)$ and $\widetilde{\Delta}$ is one of its singular perturbations, i.e., such that the set $\left\{u \in H^{2}\left(\mathbb{R}^{3}\right) \cap \operatorname{dom}(\widetilde{\Delta}): \Delta u=\widetilde{\Delta} u\right\}$ is dense. Typically $\widetilde{\Delta}$ corresponds to a self-adjoint realization of the Laplace operator with some kind of boundary conditions imposed on a null subset; in particular our results apply to standard, either separating or semi-transparent, boundary conditions at $\Gamma=\partial \Omega$, where $\Omega \subset \mathbb{R}^{3}$ is a bounded Lipschitz domain. Similar results hold in the case the boundary conditions are assigned only on $\Sigma \subset \Gamma$, a relatively open subset with a Lipschitz boundary. We show that either the obstacle $\Omega$ or the screen $\Sigma$ are determined by the knowledge of the Scattering Matrix, equivalently of the Far Field Operator, at a single frequency.
\end{abstract}

\section{INTRODUCTION}

In the recent paper [22] (also see [24] for the case of smooth boundaries and [5] for similar results in the case of smooth boundaries and under additional trace-class conditions) we obtained a representation formula for the scattering matrix $S_{\lambda}^{\Lambda}: L^{2}\left(\mathbb{S}^{2}\right) \rightarrow L^{2}\left(\mathbb{S}^{2}\right)$ relative to the scattering couple $\left(\Delta, \Delta_{\Lambda}\right)$, where $\Delta$ is the self-adjoint free Laplacian in $L^{2}\left(\mathbb{R}^{3}\right)$ and $\Delta_{\Lambda}$ is a self-adjoint realization of the Laplacian with boundary conditions at $\Gamma$, the Lipschitz boundary of the bounded domain $\Omega \subset \mathbb{R}^{3}$. Here $\Lambda: z \mapsto \Lambda_{z}$ is an operator-valued map which univocally defines $\Delta_{\Lambda}$ and fixes the boundary conditions realized by the corresponding operator (see Sections 4.1 and 5.1 below for various explicit examples). Our representation formula gives $S_{\lambda}^{\Lambda}=1-2 \pi i L_{\lambda} \Lambda_{\lambda}^{+} L_{\lambda}^{*}$, where $\Lambda_{\lambda}^{+}$is the limit of $\Lambda_{\lambda+i \epsilon}$ as $\epsilon \downarrow 0$ (which, under suitable hypotheses, exists in operator norm through a Limiting Absorption Principle, see [22]), and $L_{\lambda}$ is defined in term of the trace (either Dirichlet or Neumann or both) at the boundary $\Gamma$ of the free waves with wavenumber $|\lambda|^{1 / 2}$. Introducing the Far-Field operator $F_{\lambda}^{\Lambda}:=\frac{1}{2 \pi i}\left(1-S_{\lambda}^{\Lambda}\right)$ (see [18, relation (1.31)]), one gets $F_{\lambda}^{\Lambda}=L_{\lambda} \Lambda_{\lambda}^{+} L_{\lambda}^{*}$; such a factorized form suggests to study the inverse scattering problem (concerning the reconstruction of the shape of $\Omega$ by the knowledge of the scattering data at a fixed frequency) by means of Kirsch's Factorization Method (see [18] and references therein). Our result is the following (see Theorem 4.14 for the complete statement): let $\Lambda_{\lambda}^{+}=\left(M_{\lambda}^{+}\right)^{-1}$, where the bounded bijective operator $M_{\lambda}^{+}$has the decomposition $M_{\lambda}^{+}=M_{\circ}+K_{\lambda}, M_{\circ}$ sign-definite and $K_{\lambda}$ compact; then

$$
x \in \Omega \Longleftrightarrow \inf _{\substack{\psi \in L^{2}\left(\mathbb{S}^{2}\right) \\\left\langle\psi, \phi_{\lambda}^{x}\right\rangle_{L^{2}\left(\mathbb{S}^{2}\right)}=1}}\left|\left\langle\psi, F_{\lambda}^{\Lambda} \psi\right\rangle_{L^{2}\left(\mathbb{S}^{2}\right)}\right|>0 \Longleftrightarrow \sum_{k=1}^{\infty} \frac{\left|\left\langle\phi_{\lambda}^{x}, \psi_{\lambda, k}^{\Lambda}\right\rangle_{L^{2}\left(\mathbb{S}^{2}\right)}\right|^{2}}{\left|z_{\lambda, k}^{\Lambda}\right|}<+\infty
$$


where $\phi_{\lambda}^{x}(\xi):=e^{i|\lambda|^{1 / 2} \xi \cdot x}$ and the sequences $\left\{z_{\lambda, k}^{\Lambda}\right\}_{1}^{\infty} \subset \mathbb{C} \backslash\{0\}$ and $\left\{\psi_{\lambda, k}^{\Lambda}\right\}_{1}^{\infty} \subset L^{2}\left(\mathbb{S}^{2}\right)$ provide the spectral resolution of the compact normal operator $F_{\lambda}^{\Lambda}$. While such a result conforms to the standard ones (the inf-criterion and the $\left(F^{*} F\right)^{1 / 4}$-method) provided in [18, Section 1.4], its advantage is due to the fact that we use a factorization where all the informations regarding the boundary conditions are encoded in the operator $\Lambda_{\lambda}^{+}$, whereas $L_{\lambda}$, for which one needs to characterize the range, is model-independent; this enhances the flexibility of our approach. Moreover, with a minimal effort (which in essence consists in compressing the operator $\Lambda_{z}$ onto subspaces of functions with supports contained in $\Sigma \subset \Gamma$ ) one gets similar results in the case the boundary conditions are imposed not on the whole $\Gamma$ but only on a relatively open subset $\Sigma$ with a Lipschitz boundary. In this case the result is of the same kind, only the family of testing functions changes (see Theorem 5.6 for the precise statement): let $\Sigma_{\circ} \subset \Gamma_{\circ}, \Gamma_{\circ}$ a Lipschitz boundary; then

$$
\Sigma_{\circ} \subset \Sigma \Longleftrightarrow \inf _{\substack{\psi \in L^{2}\left(\mathbb{S}^{2}\right) \\\left\langle\psi, \phi_{\lambda}^{\Sigma_{\circ}}\right\rangle_{L^{2}\left(\mathbb{S}^{2}\right)}=1}}\left|\left\langle\psi, F_{\lambda}^{\Lambda} \psi\right\rangle_{L^{2}\left(\mathbb{S}^{2}\right)}\right|>0 \Longleftrightarrow \sum_{k=1}^{\infty} \frac{\left|\left\langle\phi_{\lambda}^{\Sigma_{\circ}}, \psi_{\lambda, k}^{\Lambda}\right\rangle_{L^{2}\left(\mathbb{S}^{2}\right)}\right|^{2}}{\left|z_{\lambda, k}^{\Lambda}\right|}+\infty,
$$

where $\phi_{\lambda}^{\Sigma_{\circ}}(\xi):=\int_{\Sigma_{\circ}} \phi_{\lambda}^{x}(\xi) d \sigma_{\Gamma_{\circ}}(x)$.

We provide several examples where Theorems 4.14 and5.6 apply. In particular, we consider obstacles and screens reconstruction for the following boundary conditions:

- Dirichlet $\gamma_{0} u=0$ (see Subsections 4.1.1 and 5.1.1);

- Neumann $\gamma_{1} u=0$ (see Subsections 4.1.2 and 5.1.2);

- semi-transparent

$$
\left\{\begin{array}{l}
\alpha \gamma_{0} u=\left[\gamma_{1}\right] u \\
{\left[\gamma_{0}\right] u=0}
\end{array}\right.
$$

either $\alpha>0$ or $\alpha<0$ (see Subsections 4.1.3 and 5.1.3);

- semi-transparent

$$
\left\{\begin{array}{l}
\gamma_{1} u=\theta\left[\gamma_{0}\right] u \\
{\left[\gamma_{1}\right] u=0}
\end{array}\right.
$$

$\theta$ real-valued (see Subsections 4.1 .4 and 5.1.4);

- local of the kind

$$
\left\{\begin{array}{l}
\gamma_{0} u=b_{11}\left[\gamma_{0}\right] u+b_{12}\left[\gamma_{1}\right] u \\
\gamma_{1} u=b_{12}^{*}\left[\gamma_{0}\right] u+b_{22}\left[\gamma_{1}\right] u
\end{array}\right.
$$

$b_{11}<0, b_{22}$ real-valued (see Subsections 4.1.5 and 5.1.5).

A huge literature is devoted to obstacle reconstruction from scattering data; we just recall some papers where the Factorization Method is used in connection with the models here treated. Dirichlet and Neumann obstacles have been considered in [17] (see also [18, Chap. 1]); Dirichlet screens have been studied firstly, in a 2-dimensional setting, in [20]. Semitransparent interface conditions appear, apart in quantum mechanical models (see, e.g., 8], [4] and references therein), in connections with acoustic models with gradient singularities, see [25]. Conditions of the type $\alpha \gamma_{0}=\left[\gamma_{1}\right] u$ appear in [19] and [6] in a non self-adjoint setting (i.e. when $\alpha$ is complex-valued): this compels the use of different data operators. An appropriate choice of the functions $b_{i j}$ in (1.1) gives the classical Robin boundary conditions; 
the latter have been considered in [11] (see also [18, Chap. 2]) and [7]. In these papers, as in the previous case, a non self-adjoint setting is used and different data operators enters in the reconstruction formulae.

In this paper, as regards scattering, we use a quantum mechanics point of view (see Section 3.2); however, as recalled in Section 3.3 below (see also [35] for the case of Neumann boundary conditions), the scattering theory for Schrödinger-type equations is equivalent to the one for wave-type equations. Hence our reconstruction results apply to diffusions of both classical and quantum waves.

In order to simplify the exposition, our results are stated in dimension $d=3$; however they hold in any dimension $d \geq 2$. Finally, we presume that, by the same techniques, our approach can be extended to the case in which the Laplace operator is replaced by a more general 2nd order elliptic differential operator.

Acknowledgements. The authors are indebted to Mourad Sini for the fruitful discussions which largely inspired this work.

\section{Notations AND PRELIMINARIES.}

\subsection{Notations.}

- $\|\cdot\|_{\mathbf{X}}$ denotes the norm on the complex Banach space $\mathbf{X}$; in case $\mathbf{X}$ is a Hilbert space, $\langle\cdot, \cdot\rangle_{\mathbf{X}}$ denotes the (conjugate-linear w.r.t. the first argument) scalar product.

- $\langle\cdot, \cdot\rangle_{\mathbf{X}^{*}, \mathbf{X}}$ denotes the duality (assumed to be conjugate-linear w.r.t. the first argument) between the dual couple $\left(\mathrm{X}^{*}, \mathrm{X}\right)$.

- $L^{*}: \operatorname{dom}\left(L^{*}\right) \subseteq \mathrm{Y}^{*} \rightarrow \mathrm{X}^{*}$ denotes the dual of the densely defined linear operator $L$ : $\operatorname{dom}(L) \subseteq \mathrm{X} \rightarrow \mathrm{Y}$; in a Hilbert spaces setting $L^{*}$ denotes the adjoint operator.

- $\rho(A)$ and $\sigma(A)$ denote the resolvent set and the spectrum of the self-adjoint operator $A$; $\sigma_{\mathrm{p}}(A), \sigma_{\mathrm{pp}}(A), \sigma_{\mathrm{ac}}(A), \sigma_{\mathrm{sc}}(A), \sigma_{\mathrm{ess}}(A), \sigma_{\mathrm{disc}}(A)$, denote the point, pure point, absolutely continuous, singular continuous, essential and discrete spectra.

- $\mathscr{B}(\mathrm{X}, \mathrm{Y}), \mathscr{B}(\mathrm{X}) \equiv \mathscr{B}(\mathrm{X}, \mathrm{X})$, denote the Banach space of bounded linear operator on the Banach space $X$ to the Banach space $Y ;\|\cdot\|_{X, Y}$ denotes the corresponding norm.

- $\mathrm{X} \hookrightarrow \mathrm{Y}$ means that $\mathrm{X} \subseteq \mathrm{Y}$ and for any $u \in \mathrm{X}$ there exists $c>0$ such that $\|u\|_{\mathrm{Y}} \leq c\|u\|_{\mathrm{X}}$; we say that $X$ is continuously embedded into $Y$.

- $u \mid \Gamma$ denotes the restriction of the function $u$ to the set $\Gamma ; L \mid \mathrm{V}$ denotes the restriction of the linear operator $L$ to the subspace $\mathrm{V}$.

- $H^{s}\left(\mathbb{R}^{3}\right), s \in \mathbb{R}$, denotes the scale of Hilbert space of Sobolev functions on $\mathbb{R}^{3}$, i.e. $u \in$ $H^{s}\left(\mathbb{R}^{3}\right)$ if and only if $k \mapsto\left(1+\|k\|^{2}\right)^{s / 2} \widehat{u}(k)$ is square integrable, $\widehat{u}$ denoting Fourier transform.

- $\Omega \equiv \Omega_{\text {in }} \subset \mathbb{R}^{3}$ denotes a bounded open set with a Lipschitz boundary $\Gamma ; \Omega_{\text {ex }}:=\mathbb{R}^{3} \backslash \bar{\Omega}$.

- $\gamma_{0}$ and $\gamma_{1}$ denote the Dirichlet and Neumann traces on the boundary $\Gamma$.

- $\Delta_{\Omega_{\text {in/ex }}}^{D}$ denotes the self-adjoint operator in $L^{2}\left(\Omega_{\text {in/ex }}\right)$ representing the Laplace operator with homogeneous Dirichlet boundary conditions at $\Gamma$. 
- $\Delta_{\Omega_{\text {in } / \text { ex }}^{N}}^{N}$ denotes the self-adjoint operator in $L^{2}\left(\Omega_{\text {in/ex }}\right)$ representing the Laplace operator with homogeneous Neumann boundary conditions at $\Gamma$.

- $H^{s}\left(\Omega_{\mathrm{in} / \mathrm{ex}}\right), s \in \mathbb{R}$, denotes the scale of Hilbert space of Sobolev functions on $\Omega_{\mathrm{in} / \mathrm{ex}}$.

- $\mathscr{C}^{\kappa}(\Gamma)$ denotes the space of Hölder-continuous functions of order $\kappa$ on $\Gamma$.

- $H^{s}(\Gamma),|s| \leq 1$, denotes the Hilbert space of Sobolev functions of order $s$ on $\Gamma$.

- $\mathscr{M}\left(H^{s}(\Gamma), H^{t}(\Gamma)\right), \mathscr{M}\left(H^{s}(\Gamma), H^{s}(\Gamma)\right) \equiv \mathscr{M}\left(H^{s}(\Gamma)\right)$, denotes the space of Sobolev multipliers from $H^{s}(\Gamma)$ to $H^{t}(\Gamma)$.

- $s_{\sharp}, \sharp=D, N$, denote the indices $s_{D}=1 / 2, s_{N}=-1 / 2$.

- $\varphi_{n} \rightarrow \varphi$ means that the sequence $\left\{\varphi_{n}\right\}_{1}^{\infty}$ weakly converges to $\varphi$.

- $\mathrm{V}^{\perp} \subseteq \mathrm{X}^{*}$, denotes the annihilator $\mathrm{V}^{\perp}=\left\{x^{*} \in \mathrm{X}^{*}:\left\langle x^{*}, x\right\rangle_{\mathrm{X}^{*}, \mathrm{X}}=0\right.$ for all $\left.x \in \mathrm{V}\right\}$ of the subspace $\mathrm{V} \subseteq \mathrm{X}$.

2.2. Trace maps and layer operators on Lipschitz manifolds. Let $\Gamma$ be the compact Lipschitz manifold given by the boundary of $\Omega \subset \mathbb{R}^{3}$. Let $\gamma_{0}$ be the map defined by the restriction of $u \in \mathscr{C}_{\text {comp }}^{\infty}\left(\mathbb{R}^{3}\right)$ along the set $\Gamma: \gamma_{0} u:=u \mid \Gamma$. Then, by [13, Theorem 1, Chapter VII], such a map has a bounded and surjective extension to $H^{s+1 / 2}\left(\mathbb{R}^{3}\right)$ for any $s>0$ :

$$
\gamma_{0}: H^{s+1 / 2}\left(\mathbb{R}^{3}\right) \rightarrow B_{2,2}^{s}(\Gamma) .
$$

Here the Hilbert space $B_{2,2}^{s}(\Gamma)$ is a Besov-like space (see [13, Section 2, Chapter V] for the precise definitions); $B_{2,2}^{s}(\Gamma)$ identifies with $H^{s}(\Gamma)$ whenever $0<s<1$ (see [13, Section 1.1, chap. V]), where $H^{s}(\Gamma)$ denotes the usual fractional Sobolev space on $\Gamma$ (see e.g. [26, Chapter 3]). If $\Gamma$ is a manifold of class $\mathscr{C}^{\kappa, 1}, \kappa \geq 0$, then $B_{2,2}^{s}(\Gamma)=H^{s}(\Gamma)$ for any $s \leq \kappa+1$. We use the following notations for the dual (with respect to the $L^{2}(\Gamma)$-pairing) spaces: $\left(B_{2,2}^{s}(\Gamma)\right)^{*} \equiv B_{2,2}^{-s}(\Gamma)$.

By [33, Proposition 20.5], the embeddings $B_{2,2}^{s_{2}}(\Gamma) \hookrightarrow B_{2,2}^{s_{1}}(\Gamma), s_{2}>s_{1}$, and $B_{2,2}^{s}(\Gamma) \hookrightarrow$ $L^{2 /(1-s)}(\Gamma), 0<s<1$, are compact.

Let $\Delta: H^{s+2}\left(\mathbb{R}^{3}\right) \rightarrow H^{s}\left(\mathbb{R}^{3}\right)$ be the distributional Laplacian; in the following the resolvent $R_{z}^{0} \equiv(-\Delta+z)^{-1}, z \in \mathbb{C} \backslash(-\infty, 0]$, is viewed as a map in $\mathscr{B}\left(H^{s}\left(\mathbb{R}^{n}\right), H^{s+2}\left(\mathbb{R}^{n}\right)\right), s \in \mathbb{R}$. Given $s>0$, by the mapping properties (2.1) one gets, for the dual of the trace map,

$$
\gamma_{0}^{*}: B_{2,2}^{-s}(\Gamma) \rightarrow H^{-s-1 / 2}\left(\mathbb{R}^{3}\right)
$$

and so we can define the bounded operator (the single-layer potential)

$$
S L_{z}:=R_{z}^{0} \gamma_{0}^{*}: B_{2,2}^{-s}(\Gamma) \rightarrow H^{3 / 2-s}\left(\mathbb{R}^{3}\right) .
$$

By resolvent identity one has

$$
S L_{z}-S L_{w}=(w-z) R_{z}^{0} S L_{w} .
$$

By (2.1) and (2.2), one obtains the bounded operator

$$
\gamma_{0} S L_{z}: B_{2,2}^{-s}(\Gamma) \rightarrow B_{2,2}^{1-s}(\Gamma) .
$$

In the following $\Delta_{\Omega_{\text {in } / \mathrm{ex}}}$ denote the distributional Laplacians on $\Omega_{\text {in/ex }}$. 
The one-sided, zero and first order, trace operators $\gamma_{0}^{\text {in/ex }}$ and $\gamma_{1}^{\text {in/ex }}=\nu \cdot \gamma_{0}^{\text {in/ex }} \nabla(\nu$ denoting the outward normal vector at the boundary) defined on smooth functions in $\mathcal{C}_{\text {comp }}^{\infty}\left(\bar{\Omega}_{\text {in/ex }}\right)$ extend to bounded and surjective linear operators (see e.g. [26, Theorem 3.38])

$$
\gamma_{0}^{\text {in } / \text { ex }} \in \mathscr{B}\left(H^{s+1 / 2}\left(\Omega_{\text {in } / \mathrm{ex}}\right), H^{s}(\Gamma)\right), \quad 0<s<1 .
$$

and

$$
\gamma_{1}^{\text {in } / \mathrm{ex}} \in \mathscr{B}\left(H^{s+3 / 2}\left(\Omega_{\text {in } / \mathrm{ex}}\right), H^{s}(\Gamma)\right), \quad 0<s<1
$$

(we refer to [26, Chapter 3] for the definition of the Sobolev spaces $H^{s}\left(\Omega_{\text {in/ex }}\right)$ and $H^{s}(\Gamma)$ ). Using these maps and setting $H^{s}\left(\mathbb{R}^{3} \backslash \Gamma\right):=H^{s}\left(\Omega_{\text {in }}\right) \oplus H^{s}\left(\Omega_{\mathrm{ex}}\right)$, the two-sided bounded and surjective trace operators are defined according to

$$
\begin{aligned}
& \gamma_{0}: H^{s+1 / 2}\left(\mathbb{R}^{3} \backslash \Gamma\right) \rightarrow H^{s}(\Gamma), \quad \gamma_{0}\left(u_{\mathrm{in}} \oplus u_{\mathrm{ex}}\right):=\frac{1}{2}\left(\gamma_{0}^{\mathrm{in}} u_{\mathrm{in}}+\gamma_{0}^{\mathrm{ex}} u_{\mathrm{ex}}\right), \\
& \gamma_{1}: H^{s+3 / 2}\left(\mathbb{R}^{3} \backslash \Gamma\right) \rightarrow H^{s}(\Gamma), \quad \gamma_{1}\left(u_{\mathrm{in}} \oplus u_{\mathrm{ex}}\right):=\frac{1}{2}\left(\gamma_{1}^{\mathrm{in}} u_{\mathrm{in}}+\gamma_{0}^{\mathrm{ex}} u_{\mathrm{ex}}\right),
\end{aligned}
$$

while the corresponding jumps are

$$
\begin{aligned}
& {\left[\gamma_{0}\right]: H^{s+1 / 2}\left(\mathbb{R}^{3} \backslash \Gamma\right) \rightarrow H^{s}(\Gamma), \quad\left[\gamma_{0}\right]\left(u_{\text {in }} \oplus u_{\mathrm{ex}}\right):=\gamma_{0}^{\mathrm{in}} u_{\mathrm{in}}-\gamma_{0}^{\mathrm{ex}} u_{\mathrm{ex}}} \\
& {\left[\gamma_{1}\right]: H^{s+3 / 2}\left(\mathbb{R}^{3} \backslash \Gamma\right) \rightarrow H^{s}(\Gamma), \quad\left[\gamma_{1}\right]\left(u_{\mathrm{in}} \oplus u_{\mathrm{ex}}\right):=\gamma_{1}^{\mathrm{in}} u_{\mathrm{in}}-\gamma_{1}^{\mathrm{ex}} u_{\mathrm{ex}} .}
\end{aligned}
$$

Let us notice that in the case $u=u_{\text {in }} \oplus u_{\text {ex }} \in H^{s+1 / 2}\left(\mathbb{R}^{n}\right), 0<s<1, \gamma_{0}$ in (2.6) coincides with the map defined in (2.1) and so there is no ambiguity in our notations; this also entails that $\gamma_{0}$ remains surjective even if restricted to $H^{2}\left(\mathbb{R}^{3}\right)$. Similarly the map $\gamma_{1}$ is surjective onto $H^{s}(\Gamma)$ even if restricted to $H^{s+3 / 2}\left(\mathbb{R}^{3}\right)$.

By [26, Lemma 4.3], the trace maps $\gamma_{1}^{\text {in/ex }}$ can be extended to the spaces

$$
\begin{aligned}
H_{\Delta}^{1}\left(\Omega_{\text {in } / \mathrm{ex}}\right):=\{ & \left.u_{\text {in } / \mathrm{ex}} \in H^{1}\left(\Omega_{\text {in } / \mathrm{ex}}\right): \Delta_{\Omega_{\text {in } / \mathrm{ex}}} u_{\text {in/ex }} \in L^{2}\left(\Omega_{\text {in } / \mathrm{ex}}\right)\right\}: \\
& \gamma_{1}^{\text {in } / \mathrm{ex}}: H_{\Delta}^{1}\left(\Omega_{\text {in } / \mathrm{ex}}\right) \rightarrow H^{-1 / 2}(\Gamma) .
\end{aligned}
$$

This gives the analogous extensions of the maps $\gamma_{1}$ and $\left[\gamma_{1}\right]$ defined on $H_{\Delta}^{1}\left(\mathbb{R}^{3} \backslash \Gamma\right):=$ $H_{\Delta}^{1}\left(\Omega_{\mathrm{in}}\right) \oplus H_{\Delta}^{1}\left(\Omega_{\mathrm{ex}}\right)$ with values in $H^{-1 / 2}(\Gamma)$.

By using a cut-off function $\chi \in \mathcal{C}_{\text {comp }}^{\infty}\left(\mathbb{R}^{n}\right)$ such that $\chi=1$ in a neighborhood of $\Omega_{\text {in }}$, all the maps defined above can be extended (and we use the same notation) to functions $u$ such that $\chi u$ is in the right function space.

The single-layer operator $S L_{z}$ has been already introduced above; now we recall the definition of double-layer operator $D L_{z}, z \in \mathbb{C} \backslash(-\infty, 0]$ : by the dual map

$$
\gamma_{1}^{*}: H^{-s}(\Gamma) \rightarrow H^{-s-3 / 2}\left(\mathbb{R}^{3}\right)
$$

and by the resolvent $R_{z}^{0} \in \mathscr{B}\left(H^{s}\left(\mathbb{R}^{3}\right), H^{s+2}\left(\mathbb{R}^{3}\right)\right)$, one defines the bounded operator

$$
D L_{z}: H^{-s}(\Gamma) \rightarrow H^{-s+1 / 2}\left(\mathbb{R}^{3}\right), \quad D L_{z}:=R_{z}^{0}\left(\gamma_{1}\right)^{*}, \quad 0<s<1 .
$$

By resolvent identity one has

$$
D L_{z}-D L_{w}=(z-w) R_{z}^{0} D L_{w} .
$$


By the mapping properties of the layer operators, one gets (see [26, Theorem 6.11])

$$
\chi S L_{z} \in \mathscr{B}\left(H^{-1 / 2}(\Gamma), H^{1}\left(\mathbb{R}^{3}\right)\right), \quad \chi D L_{z} \in \mathscr{B}\left(H^{1 / 2}(\Gamma), H^{1}\left(\mathbb{R}^{3} \backslash \Gamma\right)\right),
$$

for any $\chi \in \mathscr{C}_{\text {comp }}^{\infty}\left(\mathbb{R}^{3}\right)$; by $\left(-\left(\Delta_{\Omega_{\mathrm{in}}} \oplus \Delta_{\Omega_{\mathrm{ex}}}\right)+z\right) S L_{z} \phi=\left(-\left(\Delta_{\Omega_{\mathrm{in}}} \oplus \Delta_{\Omega_{\mathrm{ex}}}\right)+z\right) D L_{z} \varphi=0$, one gets $\chi S L_{z} \phi \in H_{\Delta}^{1}\left(\mathbb{R}^{n} \backslash \Gamma\right), \phi \in H^{-1 / 2}(\Gamma)$, and $\chi D L_{z} \varphi \in H_{\Delta}^{1}\left(\mathbb{R}^{n} \backslash \Gamma\right), \varphi \in H^{1 / 2}(\Gamma)$. Thus

$$
\gamma_{0} S L_{z} \in \mathscr{B}\left(H^{-1 / 2}(\Gamma), H^{1 / 2}(\Gamma)\right), \quad \gamma_{1} D L_{z} \in \mathscr{B}\left(H^{1 / 2}(\Gamma), H^{-1 / 2}(\Gamma)\right) .
$$

These mapping properties can be extended to a larger range of Sobolev spaces (see, e.g., [26, Theorem 6.12 and successive remarks]):

$\gamma_{0} S L_{z} \in \mathscr{B}\left(H^{s-1 / 2}(\Gamma), H^{s+1 / 2}(\Gamma)\right), \quad \gamma_{1} D L_{z} \in \mathscr{B}\left(H^{s+1 / 2}(\Gamma), H^{s-1 / 2}(\Gamma)\right), \quad-1 / 2 \leq s \leq 1 / 2$.

By the Limiting Absorption Principle for the free Laplacian (see, e.g., [21, Section 18]), duality and interpolation, one has that the limits

$$
R_{\lambda}^{0, \pm}:=\lim _{\epsilon \downarrow 0} R_{\lambda \pm i \epsilon}^{0}
$$

exist in $\in \mathscr{B}\left(H_{w}^{-s}\left(\mathbb{R}^{3}\right), H_{-w}^{-s+2}\left(\mathbb{R}^{3}\right)\right), w>1 / 2,0 \leq s \leq 2$ (here $H_{w}^{s}\left(\mathbb{R}^{3}\right)$ denotes the weighted Sobolev space of order $s$ with weight $\left.\varphi(x)=\left(1+\|x\|^{2}\right)^{w / 2}\right)$. Thus, since $\Gamma$ is bounded, the limits

$$
S L_{\lambda}^{ \pm}:=R_{\lambda}^{0, \pm} \gamma_{0}^{*}=\lim _{\epsilon \downarrow 0} S L_{\lambda \pm i \epsilon}, \quad D L_{\lambda}^{ \pm}:=R_{\lambda}^{0, \pm} \gamma_{1}^{*}=\lim _{\epsilon \downarrow 0} D L_{\lambda \pm i \epsilon}
$$

exist in $\mathscr{B}\left(B_{2,2}^{-s}(\Gamma), H_{-w}^{3 / 2-s}\left(\mathbb{R}^{3}\right)\right), 0<s \leq 3 / 2$, and $\mathscr{B}\left(H^{-s}(\Gamma), H_{-w}^{1 / 2-s}\left(\mathbb{R}^{3}\right)\right), 0<s \leq 1 / 2$, respectively. Moreover, by the identities (2.3), (2.11) and by $S L_{z} \in \mathscr{B}\left(B_{2,2}^{-3 / 2}(\Gamma), L_{w}^{2}\left(\mathbb{R}^{n}\right)\right)$, $D L_{z} \in \mathscr{B}\left(H^{-1 / 2}(\Gamma), L_{w}^{2}\left(\mathbb{R}^{n}\right)\right)$ (see [24, relation (4.10)]) one has

$$
S L_{\lambda}^{ \pm}=S L_{z}+(z-\lambda) R_{\lambda}^{0, \pm} S L_{z}, \quad D L_{\lambda}^{ \pm}=D L_{z}+(z-\lambda) R_{\lambda}^{0, \pm} D L_{z} .
$$

\section{Direct Scattering Theory for Singular Perturbations.}

3.1. Singular Perturbations of the Laplace operator. Let $\Delta: H^{2}\left(\mathbb{R}^{3}\right) \subseteq L^{2}\left(\mathbb{R}^{3}\right) \rightarrow$ $L^{2}\left(\mathbb{R}^{3}\right)$ be the self-adjoint operator given by the free Laplacian on the whole space. Another self-adjoint operator $\widetilde{\Delta}: \operatorname{dom}(\widetilde{\Delta}) \subseteq L^{2}\left(\mathbb{R}^{3}\right) \rightarrow L^{2}\left(\mathbb{R}^{3}\right)$ is said to be a singular perturbation of $\Delta$ if the set

$$
\mathrm{D}:=\left\{u \in H^{2}\left(\mathbb{R}^{3}\right) \cap \operatorname{dom}(\widetilde{\Delta}): \Delta u=\widetilde{\Delta} u\right\}
$$

is dense in $L^{2}\left(\mathbb{R}^{3}\right)$. Our aim is the study of direct and inverse scattering for the couple $(\widetilde{\Delta}, \Delta)$. Notice that $\widetilde{\Delta}$ is a self-adjoint extension of the symmetric operator $\Delta^{\circ}:=\Delta|D \equiv \widetilde{\Delta}| D$; in typical situations $\widetilde{\Delta}$ represents the Laplace operator with some kind of boundary condition holding on a null subset.

3.2. Wave Operators. Given the two self-adjoint operators $\Delta$ and $\widetilde{\Delta}$, let $e^{i t \Delta}$ and $e^{i t \widetilde{\Delta}}$ be the corresponding unitary groups of evolution providing solutions of the Cauchy problems for the Schrödinger equations

$$
i \frac{d u}{d t}=-\Delta u, \quad i \frac{d u}{d t}=-\widetilde{\Delta} u .
$$


As usual in Quantum Mechanics (see, e.g., [31]), we define the Wave Operators for the scattering couple $(\widetilde{\Delta}, \Delta)$ as

$$
W_{ \pm}(\widetilde{\Delta}, \Delta) u:=\lim _{t \rightarrow \mp \infty} e^{-i t \widetilde{\Delta}} e^{i t \Delta} u
$$

One says that $W_{ \pm}(\widetilde{\Delta}, \Delta)$ exist whenever the limits exist for any vector $u \in L^{2}\left(\mathbb{R}^{3}\right)$ and then that are complete whenever

$$
\operatorname{ran}\left(W_{+}(\widetilde{\Delta}, \Delta)\right)=: \mathrm{H}_{\mathrm{in}}=\mathrm{H}_{\text {out }}:=\operatorname{ran}\left(W_{-}(\widetilde{\Delta}, \Delta)\right)=L^{2}\left(\mathbb{R}^{3}\right)_{\mathrm{ac}},
$$

where $L^{2}\left(\mathbb{R}^{3}\right)_{\text {ac }}$ denotes the absolutely continuous subspace of $\widetilde{\Delta}$. It is known that the existence of both the wave operators $W_{ \pm}(\widetilde{\Delta}, \Delta)$ and $W_{ \pm}(\Delta, \widetilde{\Delta})$ gives completeness. From the point of view of physical interpretation, a more relevant definition is the following: $W_{ \pm}(\widetilde{\Delta}, \Delta)$ are said to be asymptotically complete whenever they are complete and

$$
\mathrm{H}_{\text {in }}=\mathrm{H}_{\text {out }}=L^{2}\left(\mathbb{R}^{3}\right)_{\mathrm{pp}}^{\perp},
$$

where $L^{2}\left(\mathbb{R}^{3}\right)_{\mathrm{pp}}$ denotes the pure point subspace of $\widetilde{\Delta}$; equivalently, whenever they are complete and the singular continuous spectrum of $\widetilde{\Delta}$ is empty: $\sigma_{\mathrm{sc}}(\widetilde{\Delta})=\emptyset$. In this case $L^{2}\left(\mathbb{R}^{3}\right)$ decomposes into the direct sum of scattering states and bound states.

3.3. Scattering theory for wave equations. Suppose that $\widetilde{\Delta}$ is real (i.e., it maps realvalued functions to real-valued functions), not positive and injective (these hypotheses can be weakened, it suffices to require $\widetilde{\Delta}$ upper semi-bounded, see [15, Sections 8 and 9], [3, Section $10.3])$. Let $H_{\text {hom }}^{1}\left(\mathbb{R}^{3}\right)$ be the homogeneous Sobolev space of order one and let $\widetilde{H}_{\text {hom }}^{1}\left(\mathbb{R}^{3}\right)$ the completion, with respect to the norm $\|u\|:=\left\|(-\widetilde{\Delta})^{1 / 2} u\right\|_{L^{2}\left(\mathbb{R}^{3}\right)}$, of $\left.\operatorname{dom}(-\widetilde{\Delta})^{1 / 2}\right)$. Then the unitary group of evolutions providing the solutions of the Cauchy problems with real initial conditions

$$
\left\{\begin{array} { l } 
{ \frac { d } { d t } u ( t ) = v ( t ) } \\
{ \frac { d } { d t } v ( t ) = \Delta u ( t ) } \\
{ u ( 0 ) = u _ { 0 } \in H _ { \mathrm { hom } } ^ { 1 } ( \mathbb { R } ^ { 3 } ) } \\
{ v ( 0 ) = v _ { 0 } \in L ^ { 2 } ( \mathbb { R } ^ { 3 } ) , }
\end{array} \quad \left\{\begin{array}{l}
\frac{d}{d t} \widetilde{u}(t)=\widetilde{v}(t) \\
\frac{d}{d t} \widetilde{v}(t)=\widetilde{\Delta} \widetilde{u}(t) \\
\widetilde{u}(0)=\widetilde{u}_{0} \in \widetilde{H}_{\mathrm{hom}}^{1}\left(\mathbb{R}^{3}\right) \\
\widetilde{v}(0)=\widetilde{v}_{0} \in L^{2}\left(\mathbb{R}^{3}\right),
\end{array}\right.\right.
$$

are unitary equivalent, by the maps

$$
u \oplus v \mapsto(-\Delta)^{1 / 2} u+i v, \quad \widetilde{u} \oplus \widetilde{v} \mapsto(-\widetilde{\Delta})^{1 / 2} \widetilde{u}+i \widetilde{v},
$$

to the Schrödinger unitary groups in the complex Hilbert space $L^{2}\left(\mathbb{R}^{3}\right)$ given by $e^{-i t(-\Delta)^{1 / 2}}$ and $e^{-i t(-\widetilde{\Delta})^{1 / 2}}$ respectively. By the Kato-Birman invariance principle (see, e.g., [3, Section 11.3.3]), if both the wave operators $W_{ \pm}(\widetilde{\Delta}, \Delta)$ and $W_{ \pm}\left(-(-\widetilde{\Delta})^{1 / 2},-(-\Delta)^{1 / 2}\right)$ exist, then they are equal (by the Kato-Birman criterion, see [16, Theorem 4.8, Chapter X], equality holds whenever the difference of some power of the resolvents is trace-class; for the models discussed below this is true under some additional regularity hypotheses on $\Gamma$, see [23, Theorems 4.11 and 4.12]). In this case the scattering theory for the couple of Schrödinger equations (3.1) is equivalent to the one for the couple of wave equations

$$
\frac{d^{2} u}{d t^{2}}=\Delta u, \quad \frac{d^{2} u}{d t^{2}}=\widetilde{\Delta} u
$$


3.4. A resolvent formula for singular perturbations. Given an auxiliary Hilbert space $\mathrm{K}$, we introduce a linear application $\tau: H^{2}\left(\mathbb{R}^{3}\right) \rightarrow \mathrm{K}$ which plays the role of an abstract trace (evaluation) map. We assume that

1. $\tau$ is continuous;

2. $\tau$ is surjective (so that $\mathrm{K}$ plays the role of the trace space);

3. $\operatorname{ker}(\tau)$ is dense in $L^{2}\left(\mathbb{R}^{3}\right)$.

In the following we do not identify $\mathrm{K}$ with its dual $\mathrm{K}^{*}$; however we use $\mathrm{K}^{* *} \equiv \mathrm{K}$. Tipically $\mathrm{K} \hookrightarrow \mathrm{K}_{0} \hookrightarrow \mathrm{K}^{*}$ and the $\mathrm{K}-\mathrm{K}^{*}$ duality $\langle\cdot, \cdot\rangle_{\mathrm{K}^{*} \mathrm{~K}}$ (conjugate-linear with respect to the first variable) is defined in terms of the scalar product of the Hilbert space $\mathbf{K}_{0}$. For any $z \in \rho\left(A_{0}\right)$ we define the bounded operators

$$
R_{z}^{0}:=(-\Delta+z)^{-1}: L^{2}\left(\mathbb{R}^{3}\right) \rightarrow H^{2}\left(\mathbb{R}^{3}\right)
$$

and

$$
G_{z}:=\left(\tau R_{z^{*}}^{0}\right)^{*}: \mathrm{K}^{*} \rightarrow L^{2}\left(\mathbb{R}^{3}\right) .
$$

Then, given a reflexive Banach space $\mathrm{X}$ such that $\mathrm{K} \hookrightarrow \mathrm{X}$, we consider, for some not empty set $Z_{\Lambda} \subseteq \mathbb{C} \backslash(-\infty, 0]$ which is symmetric with respect to the real axis (i.e., $\left.z \in Z_{\Lambda} \Rightarrow z^{*} \in Z_{\Lambda}\right)$, a map

$$
\Lambda: Z_{\Lambda} \rightarrow \mathscr{B}\left(\mathrm{X}, \mathrm{X}^{*}\right), \quad z \mapsto \Lambda_{z}
$$

such that

$$
\Lambda_{z}^{*}=\Lambda_{z^{*}}, \quad \Lambda_{w}-\Lambda_{z}=(z-w) \Lambda_{w} G_{w^{*}}^{*} G_{z} \Lambda_{z} .
$$

Remark 3.1. Notice that whenever there exists a family of bijections $M_{z} \in \mathscr{B}\left(\mathrm{X}^{*}, \mathrm{X}\right)$, $z \in Z_{\Lambda}$, such that $\Lambda_{z}=M_{z}^{-1}$, then (3.3) is equivalent to

$$
M_{z}^{*}=M_{z^{*}}, \quad M_{z}-M_{w}=(z-w) G_{w^{*}}^{*} G_{z} .
$$

The following result is a useful ingredient in the successive discussion about inverse scattering:

Lemma 3.2. Let $M_{z} \in \mathscr{B}\left(\mathrm{X}^{*}, \mathrm{X}\right), z \in Z_{\Lambda}$, satisfy (3.4). Then

$$
\forall z \in Z_{\Lambda} \cap \mathbb{C} \backslash \mathbb{R}, \quad \forall \phi \in \mathrm{X}^{*} \backslash\{0\}, \quad \operatorname{Im}\left\langle\phi, M_{z} \phi\right\rangle_{\mathrm{X}^{*}, \mathrm{X}} \neq 0 .
$$

Proof. By (3.4), one has $\operatorname{Im}\left\langle\phi, M_{z} \phi\right\rangle_{\mathrm{X}^{*}, \mathrm{X}}=\operatorname{Im}(z)\left\|G_{z} \phi\right\|_{L^{2}\left(\mathbb{R}^{3}\right)}^{2}$. Since $G_{z}^{*}=\tau R_{z^{*}}^{0}$ is surjective onto $\mathrm{K}, G_{z}$ has closed range by the closed range theorem. Hence, see [16, Theorem 5.2, page 231], there exists $c>0$ such that $\left\|G_{z} \phi\right\|_{L^{2}\left(\mathbb{R}^{3}\right)}^{2} \geq c\|\phi\|_{K^{*}}^{2}$. Therefore, whenever $\operatorname{Im}(z) \neq 0$,

$$
\operatorname{Im}\left\langle\phi, M_{z} \phi\right\rangle_{X^{*}, \mathrm{X}}=0 \quad \Longrightarrow\|\phi\|_{K^{*}}=0 \quad \Longrightarrow \quad \phi=0
$$

and the proof is done.

Now we recall the key result about singular perturbations of $\Delta$ (see [27, Theorem 2.1], [28, Corollary 3.2], [29, Corollary 3.2], [22, Theorem 2.4]):

Theorem 3.3. Let $\tau$ and $\Lambda$ be as above. Then the family of bounded linear maps in $L^{2}\left(\mathbb{R}^{3}\right)$

$$
R_{z}^{\Lambda}:=R_{z}^{0}+G_{z} \Lambda_{z} G_{z^{*}}^{*}, \quad z \in Z_{\Lambda},
$$


is the resolvent of a self-adjoint operator $\Delta_{\Lambda}$ which is a singular perturbation of $\Delta$. Moreover, $\Delta_{\Lambda}$ is a self-adjoint extension of the closed symmetric operator $\Delta \mid \operatorname{ker}(\tau)$ and all its selfadjoint extensions (and any singular perturbation of $\Delta$ as well) are of this kind.

Remark 3.4. The map $\Lambda: z \mapsto \Lambda_{z}$ introduced in (3.2) and (3.3) encodes the boundary conditions that the functions belonging to the self-adjointness domain of the corresponding $\Delta_{\Lambda}$ have to satisfy. We refer to the successive Sections 4.1 and 5.1 below for various explicit examples. Notice that the properties required in (3.3) are necessary for the operator family $z \mapsto R_{z}^{\Lambda}$ in (3.5) to satisfy the first resolvent identity and $\left(R_{z}^{\Lambda}\right)^{*}=R_{z^{*}}^{\Lambda}$ (see [27, page 113]).

Then, building on some results by Schechter conceived for perturbations by a regular potential (see [30, Section 9.4]), one gets a completeness criterion for the scattering couple $\left(\Delta_{\Lambda}, \Delta\right)$ (see [22, Theorem 2.8]):

Theorem 3.5. Suppose that there exists an open subset $E \subseteq \mathbb{R}$ of full measure such that for any open and bounded $I, \bar{I} \subset E$,

$$
\sup _{(\lambda, \epsilon) \in I \times(0,1)} \epsilon^{\frac{1}{2}}\left\|G_{\lambda \pm i \epsilon}\right\|_{\mathscr{B}\left(\mathrm{K}^{*}, L^{2}\left(\mathbb{R}^{3}\right)\right)}<+\infty,
$$

and

$$
\sup _{(\lambda, \epsilon) \in I \times(0,1)}\left\|\Lambda_{\lambda \pm i \epsilon}\right\|_{\mathscr{B}\left(\mathrm{K}, \mathrm{K}^{*}\right)}<+\infty .
$$

Then both the wave operators $W_{ \pm}\left(\Delta_{\Lambda}, \Delta\right)$ and $W_{ \pm}\left(\Delta, \Delta_{\Lambda}\right)$ exists and are complete.

3.5. The Scattering Matrix. According to Theorem [3.3, whenever (3.6) and (3.7) hold, the scattering operator

$$
S_{\Lambda}:=W_{+}\left(\Delta_{\Lambda}, \Delta\right)^{*} W_{-}\left(\Delta_{\Lambda}, \Delta\right) .
$$

is a well defined unitary map. Given the direct integral representation of $L^{2}\left(\mathbb{R}^{3}\right)$ with respect to the spectral measure of $\Delta$, i.e. the unitary map (here $\mathbb{S}^{2}$ denotes the 2-dimensional unitary sphere in $\mathbb{R}^{3}$ )

$$
\mathcal{F}_{0}: L^{2}\left(\mathbb{R}^{3}\right) \rightarrow L^{2}\left((-\infty, 0) ; L^{2}\left(\mathbb{S}^{2}\right)\right), \quad\left(\mathcal{F}_{0} u\right)_{\lambda}(\xi)=-\frac{|\lambda|^{1 / 4}}{2^{1 / 2}} \widehat{u}\left(|\lambda|^{1 / 2} \xi\right),
$$

which diagonalizes $\Delta$, we define the scattering matrix

$$
S_{\lambda}^{\Lambda}: L^{2}\left(\mathbb{S}^{2}\right) \rightarrow L^{2}\left(\mathbb{S}^{2}\right)
$$

by the relation

$$
\mathcal{F}_{0} S_{\Lambda} \mathcal{F}_{0}^{*} u_{\lambda}=S_{\lambda}^{\Lambda} u_{\lambda}
$$

The scattering matrix is better studied using Limiting Absorption Principle and stationary scattering theory (see, e.g., [34]). However, for typical scattering couples $\left(\Delta_{\Lambda}, \Delta\right)$, the hypotheses required in [34] are not satisfied. Thus at first one considers the scattering matrix for the resolvent couple $\left(R_{\mu}^{\Lambda}, R_{\mu}^{0}\right), \mu \in \rho\left(\Delta_{\Lambda}\right) \cap(0,+\infty)$, so to exploit the factorized form of the resolvent difference $R_{\mu}^{\Lambda}-R_{\mu}^{0}$ provided by formula (3.5), and then uses the Birman-Kato invariance principle (see [22, Section 4]). At the end, one obtains the following (see [22, Theorem 5.1]; notice that in reference [22], due to a repeated misprint, the $t \rightarrow \pm \infty$ limits has to be replaced by the $t \rightarrow \mp \infty$ ones) 
Theorem 3.6. Let $\Delta_{\Lambda}$ denote the self-adjoint operator corresponding to $\Lambda=\left\{\Lambda_{z}\right\}_{z \in Z_{\Lambda}}$, $\Lambda_{z} \in \mathscr{B}\left(\mathrm{X}, \mathrm{X}^{*}\right), \mathrm{K} \hookrightarrow \mathrm{X}$. Suppose that:

$$
\Delta_{\Lambda} \text { is bounded from above; }
$$$$
\text { the embedding } \operatorname{ran}\left(\Lambda_{\lambda}\right) \hookrightarrow \mathrm{K}^{*} \text { is compact for any } \lambda \geq c_{\Lambda}>0 \text {; }
$$$$
\text { there exists } \chi \in C_{\mathrm{comp}}^{\infty}\left(\mathbb{R}^{3}\right) \text { such that } \tau u=\tau(\chi u) \text { for any } u \in H^{2}\left(\mathbb{R}^{3}\right) \text {. }
$$

Then asymptotic completeness holds for the scattering couple $\left(\Delta_{\Lambda}, \Delta\right)$. Moreover,

$$
\sigma_{\mathrm{ac}}\left(\Delta_{\Lambda}\right)=\sigma_{\mathrm{ess}}\left(\Delta_{\Lambda}\right)=(-\infty, 0], \quad \sigma_{\mathrm{sc}}\left(\Delta_{\Lambda}\right)=\emptyset,
$$

the scattering matrix $S_{\lambda}^{\Lambda}$ is given by

$$
S_{\lambda}^{\Lambda}=1-2 \pi i L_{\lambda} \Lambda_{\lambda}^{+} L_{\lambda}^{*}, \quad \lambda \in E_{\Lambda}^{-}:=(-\infty, 0) \backslash \sigma_{\mathrm{p}}^{-}\left(\Delta_{\Lambda}\right),
$$

where $\sigma_{\mathrm{p}}^{-}\left(\Delta_{\Lambda}\right):=(-\infty, 0) \cap \sigma_{\mathrm{p}}\left(\Delta_{\Lambda}\right)$ is a (possibly empty) discrete set,

$$
\Lambda_{\lambda}^{+}:=\lim _{\epsilon \downarrow 0} \Lambda_{\lambda+i \epsilon}, \quad \text { the limit existing in } \mathscr{B}\left(\mathrm{X}, \mathrm{X}^{*}\right) \text {, }
$$

and

$$
L_{\lambda}: \mathrm{X}^{*} \rightarrow L^{2}\left(\mathbb{S}^{2}\right), \quad L_{\lambda} \phi(\xi):=\frac{1}{2^{1 / 2}} \frac{|\lambda|^{1 / 4}}{(2 \pi)^{3 / 2}}\left\langle\tau\left(\chi u_{\lambda}^{\xi}\right), \phi\right\rangle_{\mathrm{X}, \mathbf{X}^{*}}, \quad \lambda \in(-\infty, 0) .
$$

Here $u_{\lambda}^{\xi}(x)=e^{i|\lambda|^{1 / 2} \xi \cdot x}$ denotes the plane wave with direction $\xi \in \mathbb{S}^{2}$ and wavenumber $|\lambda|^{1 / 2}$.

Remark 3.7. Let $\Lambda_{z}=M_{z}^{-1}$ as in Remark 3.1 and suppose that the limit $M_{\lambda}^{+}:=\lim _{\epsilon \downarrow 0} M_{\lambda+i \epsilon}$ exists in $\mathscr{B}\left(\mathrm{X}^{*}, \mathrm{X}\right)$. Then, by Theorem 3.6 , the inverse $\left(M_{\lambda}^{+}\right)^{-1}$ exists in $\mathscr{B}\left(\mathrm{X}, \mathrm{X}^{*}\right)$ and $\Lambda_{\lambda}^{+}=\left(M_{\lambda}^{+}\right)^{-1}$.

\section{InVERSE SCATTERING FOR THE LAPLACE OPERATOR WITH BOUNDARY CONDITIONS ON LIPSCHITZ SURFACES.}

With reference to Theorem 3.6 and given an open, bounded set $\Omega \equiv \Omega_{\text {in }} \subset \mathbb{R}^{3}$ with a Lipschitz boundary $\Gamma$ and such that $\Omega_{\mathrm{ex}}:=\mathbb{R}^{3} \backslash \bar{\Omega}$ is connected, we consider models where the map $\tau: H^{2}\left(\mathbb{R}^{3}\right) \rightarrow \mathrm{K}$ corresponds to one of the following three different cases:

$$
\begin{aligned}
& \tau=\gamma_{0}, \quad \mathrm{~K}=B_{2,2}^{3 / 2}(\Gamma), \quad \mathrm{X}=H^{s}(\Gamma),|s| \leq 1 ; \\
& \tau=\gamma_{1}, \quad \mathrm{~K}=H^{1 / 2}(\Gamma), \quad \mathrm{X}=H^{s}(\Gamma),-1 \leq s<1 / 2 \text {; } \\
& \text { 3) } \tau=\gamma_{0} \oplus \gamma_{1}, \quad \mathrm{~K}=B_{2,2}^{3 / 2}(\Gamma) \oplus H^{1 / 2}(\Gamma), \quad \mathrm{X}=H^{s}(\Gamma) \oplus H^{t}(\Gamma),|s| \leq 1,-1 \leq t<1 / 2 \text {. }
\end{aligned}
$$

These settings, with suitable choice of the map $\Lambda$, allow to obtain all the self-adjoint extensions of the closed symmetric operator $\Delta \mid C_{\text {comp }}^{\infty}\left(\mathbb{R}^{3} \backslash \Gamma\right)$. In particular, any self-adjoint realization of the Laplace operator with boundary conditions prescribed either on the surface $\Gamma$ or on a relatively open subset $\Sigma \subset \Gamma$ can be defined in one of the above schemes, see [23, Theorem 4.4] for the case of smooth hypersurfaces. In the present framework, Theorem 3.6 allows the boundary $\Gamma$ to be Lipschitz; in the applications we give in Sections 4.1 and 5.1 hypothesis (3.10) is always satisfied since $\Omega$ is bounded; hypotheses (3.8) and (3.9) also hold, (3.8) by a direct checking and (3.9) by compact Sobolev embeddings.

The results we provide in this section apply to the cases where the boundary conditions are assigned on the whole boundary $\Gamma$. Then $\Delta_{\Lambda}$ can be interpreted as a model either 
of an extended obstacle or of a semi-transparent interface supported on $\Gamma$, whose physical properties are encoded by $\Lambda$.

Defining the Far Field operator

$$
F_{\lambda}^{\Lambda}:=\frac{1}{2 \pi i}\left(1-S_{\lambda}^{\Lambda}\right) \equiv L_{\lambda} \Lambda_{\lambda}^{+} L_{\lambda}^{*}: L^{2}\left(\mathbb{S}^{2}\right) \rightarrow L^{2}\left(\mathbb{S}^{2}\right), \quad \lambda \in E_{\Lambda}^{-},
$$

the inverse scattering problem consists in recovering the shape of the obstacle $\Omega$ from the knowledge of $F_{\lambda}^{\Lambda}$, or, equivalently, from knowledge of the scattering matrix $S_{\lambda}^{\Lambda}$.

Notation 4.1. In the following we refer to the different settings 1) - 3) above by introducing the index $\sharp$, with $\sharp=D, N, D N$ according to the possible different choices, to label the operators

$$
L_{\lambda}^{\sharp}: \mathrm{X}_{\sharp}^{s *} \rightarrow L^{2}\left(\mathbb{S}^{2}\right),
$$

associated to one of the traces $\tau_{D}=\gamma_{0}, \tau_{N}=\gamma_{1}, \tau_{D N}=\gamma_{0} \oplus \gamma_{1}$, and the spaces $\mathbf{X}=\mathbf{X}_{\sharp}^{s}$, where

$$
\mathrm{X}_{D}^{s}:=H^{1 / 2-s}(\Gamma), \quad \mathrm{X}_{N}^{s}:=H^{-1 / 2-s}(\Gamma), \quad \mathbf{X}_{D N}^{s}:=H^{1 / 2-s}(\Gamma) \oplus H^{-s}(\Gamma), \quad 0 \leq s \leq 1 / 2 .
$$

Furthermore the adopt the short-hand notations $s_{\sharp}, \sharp=D, N$, to denote the indices $s_{D}=$ $1 / 2, s_{N}=-1 / 2$.

Remark 4.2. Since $X_{\sharp}^{0} \hookrightarrow X_{\sharp}^{s}$, and hence $X_{\sharp}^{s *} \hookrightarrow X_{\sharp}^{0^{*}}$, we do not put any index $s$ in the notation for $L_{\lambda}^{\sharp}$, since we can always suppose that $L_{\lambda}^{\sharp}$ acts on $X_{\sharp}^{0^{*}}$ and is then restricted to the proper space according to the case.

Lemma 4.3. Let $\lambda \in(-\infty, 0) \backslash \sigma_{\text {disc }}\left(\Delta_{\Omega}^{\sharp}\right), \sharp=D, N$, and set

$$
\phi_{\lambda}^{x}: \mathbb{S}^{2} \rightarrow \mathbb{C}, \quad \phi_{\lambda}^{x}(\xi):=u_{\lambda}^{\xi}(x) \equiv e^{i|\lambda|^{1 / 2} \xi \cdot x} .
$$

Then

$$
x \in \Omega \quad \Longleftrightarrow \quad \phi_{\lambda}^{x} \in \operatorname{ran}\left(L_{\lambda}^{\sharp} \mid H^{s-s_{\sharp}}(\Gamma)\right), \quad s \in[0,1 / 2], \quad s_{D}=1 / 2, \quad s_{N}=-1 / 2 .
$$

Proof. Given $\lambda \in(-\infty, 0)$, let $u_{\lambda, \phi}^{\sharp}$ be the radiating solution (i.e satisfying the Sommerfeld radiating condition) in $\Omega_{\mathrm{ex}}:=\mathbb{R}^{3} \backslash \bar{\Omega}$ of Helmholtz equation $(-\Delta+\lambda) u_{\lambda, \phi}=0$ with either Dirichlet (whenever $\sharp=D$ ) or Neumann (whenever $\sharp=N$ ) boundary condition $\phi \in H^{s_{\sharp}}(\Gamma$ ). Such a solution is unique in

$$
\begin{aligned}
& H_{\Delta, l o c}^{1}\left(\Omega_{\mathrm{ex}}\right):= \\
& \left\{u \in \mathscr{D}^{\prime}\left(\Omega_{\mathrm{ex}}\right): u_{B} \in H^{1}\left(\Omega_{\mathrm{ex}} \cap B\right), \Delta u_{B} \in L^{2}\left(\Omega_{\mathrm{ex}} \cap B\right) \text { for any open ball } B \supset \bar{\Omega}\right\},
\end{aligned}
$$

where $u_{B}:=u \mid \Omega_{\mathrm{ex}} \cap B$ (see, e.g., [26, Theorem 9.11] for the Dirichlet case and [26, Exercise 9.5] for the Neumann case). Then (see, e.g., [18, Theorem 1.4], [26, Exercise 9.4(iv)]) there exists a unique $u_{\lambda, \phi}^{\sharp, \infty} \in \mathscr{C}^{\infty}\left(\mathbb{S}^{2}\right)$ such that

$$
u_{\lambda, \phi}^{\sharp}(x)=\frac{e^{i|\lambda|^{1 / 2}\|x\|}}{4 \pi\|x\|} u_{\lambda, \phi}^{\sharp, \infty}(\hat{x})+O\left(\|x\|^{-2}\right) \quad \text { as }\|x\| \rightarrow+\infty, \text { uniformly in } \hat{x}:=x /\|x\| .
$$

This defines the data-to-pattern operator

$$
K_{\lambda}^{\sharp}: H^{s \sharp}(\Gamma) \rightarrow L^{2}\left(\mathbb{S}^{2}\right), \quad K_{\lambda}^{\sharp} \phi:=u_{\lambda, \phi}^{\sharp, \infty} .
$$


Introducing the Herglotz operators $H_{\lambda}^{\sharp}: L^{2}\left(\mathbb{S}^{2}\right) \rightarrow H^{\sharp \sharp}(\Gamma)$ defined by

$$
H_{\lambda}^{D}:=\gamma_{0} H_{\lambda}, \quad H_{\lambda}^{N}:=\gamma_{1} H_{\lambda}, \quad H_{\lambda} f(x):=\int_{\mathbb{S}^{2}} \phi_{\lambda}^{x}(\xi) f(\xi) d \sigma(\xi)
$$

one has

$$
\left\langle L_{\lambda}^{\sharp} \phi, f\right\rangle_{L^{2}\left(\mathbb{S}^{2}\right)}=\frac{1}{2^{1 / 2}} \frac{|\lambda|^{1 / 4}}{(2 \pi)^{3 / 2}}\left\langle\phi, H_{\lambda}^{\sharp} f\right\rangle_{H^{-s_{\sharp}}(\Gamma), H^{s \sharp}(\Gamma)}=\frac{1}{2^{1 / 2}} \frac{|\lambda|^{1 / 4}}{(2 \pi)^{3 / 2}}\left\langle H_{\lambda}^{\sharp *} \phi, f\right\rangle_{L^{2}\left(\mathbb{S}^{2}\right)} .
$$

Since, (see [18, proofs of Theorems 1.15 and 1.26])

$$
\left(H_{\lambda}^{D}\right)^{*}=K_{\lambda}^{D} \gamma_{0} S L_{\lambda}^{+}, \quad\left(H_{\lambda}^{N}\right)^{*}=K_{\lambda}^{N} \gamma_{1} D L_{\lambda}^{+}
$$

one gets

$$
L_{\lambda}^{D}=\frac{1}{2^{1 / 2}} \frac{|\lambda|^{1 / 4}}{(2 \pi)^{3 / 2}} K_{\lambda}^{D} \gamma_{0} S L_{\lambda}^{+}, \quad L_{\lambda}^{N}=\frac{1}{2^{1 / 2}} \frac{|\lambda|^{1 / 4}}{(2 \pi)^{3 / 2}} K_{\lambda}^{N} \gamma_{1} D L_{\lambda}^{+}
$$

Since, for any $s \in[0,1 / 2]$,

$$
\gamma_{0} S L_{\lambda}^{+}: H^{s-1 / 2}(\Gamma) \rightarrow H^{s+1 / 2}(\Gamma), \quad \lambda \in(-\infty, 0) \backslash \sigma_{\text {disc }}\left(\Delta_{\Omega}^{D}\right),
$$

and

$$
\gamma_{1} D L_{\lambda}^{+}: H^{s+1 / 2}(\Gamma) \rightarrow H^{s-1 / 2}(\Gamma), \quad \lambda \in(-\infty, 0) \backslash \sigma_{\text {disc }}\left(\Delta_{\Omega}^{N}\right),
$$

are bijections (by [22, relations (5.32) and (5.33)] and the regularity results in [10, Theorem $3]$ ), one has

$$
\operatorname{ran}\left(L_{\lambda}^{\sharp} \mid H^{s-s_{\sharp}}(\Gamma)\right)=\operatorname{ran}\left(K_{\lambda}^{\sharp} \mid H^{s+s_{\sharp}}(\Gamma)\right), \quad \lambda \in(-\infty, 0) \backslash \sigma_{\text {disc }}\left(\Delta_{\Omega}^{\sharp}\right) .
$$

Finally, by [18, Theorems 1.12 and 1.27] (it is easy to check that the proofs, there given for $s=0$, hold for any $s \in[0,1 / 2])$, one has

$$
x \in \Omega \quad \Longleftrightarrow \quad \phi_{\lambda}^{x} \in \operatorname{ran}\left(K_{\lambda}^{\sharp} \mid H^{s+s_{\sharp}}(\Gamma)\right)
$$

and the thesis is proven.

Corollary 4.4. Let $\lambda \in(-\infty, 0) \backslash\left(\sigma_{\text {disc }}\left(\Delta_{\Omega}^{D}\right) \cap \sigma_{\text {disc }}\left(\Delta_{\Omega}^{N}\right)\right)$. Then

$$
x \in \Omega \quad \Longleftrightarrow \quad \phi_{\lambda}^{x} \in \operatorname{ran}\left(L_{\lambda}^{D N} \mid H^{s-1 / 2}(\Gamma) \oplus H^{t+1 / 2}(\Gamma)\right), \quad s, t \in[0,1 / 2] .
$$

Proof. Let $\lambda \in(-\infty, 0)$. Since $(-\Delta+\lambda) S L_{\lambda}^{+}(x)=(-\Delta+\lambda) D L_{\lambda}^{+}(x)=0, x \in \Omega_{\mathrm{ex}}$, one gets the identities $K_{\lambda}^{D} \gamma_{0} S L_{\lambda}^{+}=K_{\lambda}^{N} \gamma_{1} S L_{\lambda}^{+}$and $K_{\lambda}^{D} \gamma_{0} D L_{\lambda}^{+}=K_{\lambda}^{N} \gamma_{1} D L_{\lambda}^{+}$. Thus, given $\phi \oplus \varphi \in$ $H^{s-1 / 2}(\Gamma) \oplus H^{t+1 / 2}(\Gamma)$, one has

$$
\begin{aligned}
L_{\lambda}^{D N} \phi \oplus \varphi & =\frac{1}{2^{1 / 2}} \frac{|\lambda|^{1 / 4}}{(2 \pi)^{3 / 2}}\left(K_{\lambda}^{D} \gamma_{0} S L_{\lambda}^{+} \phi+K_{\lambda}^{N} \gamma_{1} D L_{\lambda}^{+} \varphi\right) \\
& =\frac{1}{2^{1 / 2}} \frac{|\lambda|^{1 / 4}}{(2 \pi)^{3 / 2}} K_{\lambda}^{N}\left(\gamma_{1} S L_{\lambda}^{+} \phi+\gamma_{1} D L_{\lambda}^{+} \varphi\right) \\
& =\frac{1}{2^{1 / 2}} \frac{|\lambda|^{1 / 4}}{(2 \pi)^{3 / 2}} K_{\lambda}^{D}\left(\gamma_{0} S L_{\lambda}^{+} \phi+\gamma_{0} D L_{\lambda}^{+} \varphi\right) .
\end{aligned}
$$

Therefore the thesis is consequence of (4.6), (4.7) and Lemma 4.3.

Let us recall the following definitions: 
Definition 4.5. Let $\mathrm{Y}$ be a reflexive Banach space. $C \in \mathscr{B}\left(\mathrm{Y}^{*}, \mathrm{Y}\right)$ is said to be: coercive, whenever there exists $c>0$ such that

$$
\forall \varphi \in \mathrm{Y}^{*}, \quad\left|\langle\varphi, C \varphi\rangle_{\mathrm{Y}^{*}, \mathrm{Y}}\right| \geq c\|\varphi\|_{\mathrm{Y}^{*}}^{2} ;
$$

positive, whenever $C=C^{*}$ and there exists $c>0$ such that

$$
\forall \varphi \in \mathrm{Y}^{*}, \quad\langle\varphi, C \varphi\rangle_{\mathrm{Y}^{*}, \mathrm{Y}} \geq c\|\varphi\|_{\mathrm{Y}^{*}}^{2} ;
$$

sign-definite, whenever either $C$ or $-C$ is positive.

Remark 4.6. Let $C \in \mathscr{B}\left(\mathrm{Y}^{*}, \mathrm{Y}\right)$ be coercive. Then $C^{*}$ is injective and so $\operatorname{ran}(C)$ is dense by $\overline{\operatorname{ran}(C)}=\operatorname{ker}\left(C^{*}\right)^{\perp}=\mathrm{Y}$. Since (4.9) implies $\|C \varphi\|_{\mathrm{Y}} \geq c\|\varphi\|_{\mathrm{Y}^{*}}, \operatorname{ran}(C)$ is closed by [16, Theorem 5.2, page 231]. Hence $C$ is a continuous bijection and therefore $C^{-1} \in \mathscr{B}\left(\mathrm{Y}, \mathrm{Y}^{*}\right)$ by the inverse mapping theorem.

We also recall the following useful coercivity criterion (see [18, Lemma 1.17]; since our statement is slightly different from the original one, for the reader convenience we give a sketch of the proof there provided):

Lemma 4.7. Let $C \in \mathscr{B}\left(\mathrm{Y}^{*}, \mathrm{Y}\right)$ be such that $\operatorname{Im}\langle\varphi, C \varphi\rangle_{\mathrm{Y}^{*}, \mathrm{Y}} \neq 0$ for any $\varphi \in \mathrm{Y}^{*} \backslash\{0\}$. Suppose $C$ has the decomposition $C=C_{\circ}+K$, where $C_{\circ}=C_{\circ}^{*}$ is coercive and $K$ is compact. Then $C$ is coercive.

Proof. Supposing that $C$ does not satisfy (4.9), one gets a sequence $\left\{\varphi_{n}\right\}_{1}^{\infty},\left\|\varphi_{n}\right\|_{\mathrm{Y}^{*}}=1$, $\varphi_{n} \rightarrow \varphi$, such that $\left\langle\varphi_{n}, C \varphi_{n}\right\rangle_{\mathrm{Y}^{*}, \mathrm{Y}} \rightarrow 0$. Since

$$
\begin{aligned}
& \left\langle\varphi_{n}-\varphi, C_{\circ}\left(\varphi_{n}-\varphi\right)\right\rangle_{\mathrm{Y}^{*}, \mathrm{Y}}=\left\langle\varphi_{n},(C-K)\left(\varphi_{n}-\varphi\right)\right\rangle_{\mathrm{Y}^{*}, \mathrm{Y}}-\left\langle C_{\circ} \varphi, \varphi_{n}-\varphi\right\rangle_{\mathrm{Y}, \mathrm{Y}^{*}} \\
= & \left\langle\varphi_{n}, C \varphi_{n}\right\rangle_{\mathrm{Y}^{*}, \mathrm{Y}}-\left\langle\varphi_{n}, K\left(\varphi_{n}-\varphi\right)\right\rangle_{\mathrm{Y}^{*}, \mathrm{Y}}-\left\langle\varphi_{n}, C \varphi\right\rangle_{\mathrm{Y}^{*}, \mathrm{Y}}-\left\langle C_{\circ} \varphi, \varphi_{n}-\varphi\right\rangle_{\mathrm{Y}_{\Upsilon^{*}}}
\end{aligned}
$$

and $\left\|K\left(\varphi_{n}-\varphi\right)\right\|_{\mathrm{Y}} \rightarrow 0$, one gets

$$
\mathbb{R} \ni \lim _{n \rightarrow \infty}\left\langle\left(\varphi_{n}-\varphi\right), C_{\circ}\left(\varphi_{n}-\varphi\right)\right\rangle_{Y^{*}, Y}=-\langle\varphi, C \varphi\rangle_{Y^{*}, Y},
$$

i.e., $\operatorname{Im}\langle\varphi, C \varphi\rangle_{Y^{*}, Y}=0$, which gives $\varphi=0$. Thus $\varphi_{n} \rightarrow 0$ and the inequality

$$
0<c \leq\left|\left\langle\varphi_{n}, C_{\circ} \varphi_{n}\right\rangle_{Y^{*}, Y}\right| \leq\left|\left\langle\varphi_{n}, C \varphi_{n}\right\rangle_{Y^{*}, Y}\right|+\left\|K \varphi_{n}\right\|_{\mathrm{Y}}
$$

is violated for $n$ sufficiently large.

\section{Notation 4.8 .}

$$
E_{D}^{-}:=(-\infty, 0) \backslash \sigma_{\text {disc }}\left(\Delta_{\Omega}^{D}\right), \quad E_{N}^{-}:=(-\infty, 0) \backslash \sigma_{\text {disc }}\left(\Delta_{\Omega}^{N}\right), \quad E_{D N}^{-}:=E_{D}^{-} \cup E_{N}^{-} .
$$

The factorized form of the operator $F_{\lambda}^{\Lambda}$, Lemma 4.3 and Corollary 4.4 suggest to take into account Kirsch's inf-criterion:

Theorem 4.9. Let $\lambda \in E_{\sharp}^{-} \cap E_{\Lambda}^{-}, \sharp=D, N, D N$, and suppose that the Far Field Operator can be factorized as

$$
F_{\lambda}^{\Lambda}=B C B^{*},
$$

where $C \in \mathscr{B}\left(\mathrm{Y}^{*}, \mathrm{Y}\right), \mathrm{Y}$ a reflexive Banach space, is coercive and $B \in \mathscr{B}\left(\mathrm{Y}, L^{2}\left(\mathbb{S}^{2}\right)\right)$ is such that

$$
\operatorname{ran}(B)=\operatorname{ran}\left(L_{\lambda}^{\sharp} \mid \mathbf{X}_{\sharp}^{s *}\right)
$$


for some $s \in[0,1 / 2]$. Then

$$
x \in \Omega \Longleftrightarrow \inf _{\substack{\psi \in L^{2}\left(\mathbb{S}^{2}\right) \\\left\langle\psi, \phi_{\lambda}^{x}\right\rangle_{L^{2}\left(\mathbb{S}^{2}\right)}=1}}\left|\left\langle\psi, F_{\lambda}^{\Lambda} \psi\right\rangle_{L^{2}\left(\mathbb{S}^{2}\right)}\right|>0
$$

where $\phi_{\lambda}^{x}$ is defined in (4.2).

Proof. By (4.9) and by [18, Theorem 1.16], for any $\phi \in L^{2}\left(\mathbb{S}^{2}\right) \backslash\{0\}$, one has

$$
\phi \in \operatorname{ran}(B) \Longleftrightarrow \inf _{\substack{\psi \in L^{2}\left(\mathbb{S}^{2}\right) \\\langle\psi, \phi\rangle_{L^{2}\left(\mathbb{S}^{2}\right)}=1}}\left|\left\langle\psi, F_{\lambda}^{\Lambda} \psi\right\rangle_{L^{2}\left(\mathbb{S}^{2}\right)}\right|>0 .
$$

The proof is then concluded by (4.11), Lemma 4.3 and Corollary 4.4 ,

The next results is a key ingredient for obtaining a different identification criterion for the shape of $\Omega$.

Theorem 4.10. Let $\lambda \in E_{\Lambda}^{-}$. Then $F_{\lambda}^{\Lambda}$ is a normal compact operator.

Proof. Since the scattering matrix $S_{\lambda}^{\Lambda}$ is unitary,

$$
4 \pi^{2}\left(F_{\lambda}^{\Lambda}\left(F_{\lambda}^{\Lambda}\right)^{*}-\left(F_{\lambda}^{\Lambda}\right)^{*} F_{\lambda}^{\Lambda}\right)=\left(S_{\lambda}^{\Lambda}\right)^{*} S_{\lambda}^{\Lambda}-S_{\lambda}^{\Lambda}\left(S_{\lambda}^{\Lambda}\right)^{*}=1-1=0
$$

and so $F_{\lambda}^{\Lambda}$ is normal. By

$$
\nabla u_{\lambda}^{\xi}=i|\lambda|^{1 / 2} \xi u_{\lambda}^{\xi}, \quad \Delta u_{\lambda}^{\xi}=-|\lambda| u_{\lambda}^{\xi},
$$

and

$$
\begin{gathered}
\left|u_{\lambda}^{\xi_{1}}(x)-u_{\lambda}^{\xi_{2}}(x)\right|^{2}=2\left(1-\cos \left(|\lambda|^{1 / 2}\left(\xi_{1}-\xi_{2}\right) \cdot x\right)\right), \\
\left|\xi_{1} u_{\lambda}^{\xi_{1}}(x)-\xi_{2} u_{\lambda}^{\xi_{2}}(x)\right|^{2}=2\left(1-\xi_{1} \cdot \xi_{2} \cos \left(|\lambda|^{1 / 2}\left(\xi_{1}-\xi_{2}\right) \cdot x\right)\right),
\end{gathered}
$$

one gets (here the constant $c$ changes from line to line)

$$
\begin{aligned}
& \left|L_{\lambda} \phi\left(\xi_{1}\right)-L_{\lambda} \phi\left(\xi_{2}\right)\right|^{2} \leq c\|\tau\|_{\mathscr{B}\left(H^{2}\left(\mathbb{R}^{3}\right), \mathrm{X}\right)}^{2}\left\|\chi\left(u_{\lambda}^{\xi_{1}}-u_{\lambda}^{\xi_{2}}\right)\right\|_{H^{2}\left(\mathbb{R}^{3}\right)}^{2}\|\phi\|_{\mathrm{X}^{*}}^{2} \\
\leq & c\left(\left\|u_{\lambda}^{\xi_{1}}-u_{\lambda}^{\xi_{2}}\right\|_{L^{2}(\operatorname{supp}(\chi))}^{2}+\left\|\nabla\left(u_{\lambda}^{\xi_{1}}-u_{\lambda}^{\xi_{2}}\right)\right\|_{L^{2}(\operatorname{supp}(\chi))}^{2}+\left\|\Delta\left(u_{\lambda}^{\xi_{1}}-u_{\lambda}^{\xi_{2}}\right)\right\|_{L^{2}(\operatorname{supp}(\chi))}^{2}\right)\|\phi\|_{\mathrm{X}^{*}}^{2} \\
\leq & c\left(\left\|u_{\lambda}^{\xi_{1}}-u_{\lambda}^{\xi_{2}}\right\|_{L^{2}(\operatorname{supp}(\chi))}^{2}+\left\|\xi_{1} u_{\lambda}^{\xi_{1}}-\xi_{2} u_{\lambda}^{\xi_{2}}\right\|_{L^{2}(\operatorname{supp}(\chi))}^{2}\right)\|\phi\|_{\mathrm{X}^{*}}^{2} \\
\leq & c\left|\xi_{1}-\xi_{2}\right|^{2}\|\phi\|_{\mathrm{X}^{*}}^{2} \\
\leq & c \operatorname{dist}_{\mathbb{S}^{2}}^{2}\left(\xi_{1}, \xi_{2}\right)\|\phi\|_{\mathrm{X}^{*}}^{2} .
\end{aligned}
$$

Therefore $L_{\lambda}$ is a bounded map with values in the space $\operatorname{Lip}\left(\mathbb{S}^{2}\right)$ of Lipschitz functions and so $L_{\lambda}$ in Theorem 3.6 is a compact operator by the compact embedding $\operatorname{Lip}\left(\mathbb{S}^{2}\right) \hookrightarrow L^{2}\left(\mathbb{S}^{2}\right)$. In conclusion, $F_{\lambda}^{\Lambda}=L_{\lambda} \Lambda_{\lambda}^{+} L_{\lambda}^{*}$ is compact since $\Lambda_{\lambda}^{+}$is bounded.

Remark 4.11. As consequence of Theorem 4.10 (and since $1-2 \pi i F_{\lambda}^{\Lambda}$ is unitary), by spectral theory for compact normal operators (see, e.g., [14, Section 6]), one has

$$
\sigma_{\text {disc }}\left(F_{\lambda}^{\Lambda}\right)=\sigma\left(F_{\lambda}^{\Lambda}\right) \backslash\{0\}=\left\{z_{\lambda, k}^{\Lambda}\right\}_{1}^{\infty} \subset\left\{z \in \mathbb{C} \backslash\{0\}:\left|z-\frac{1}{2 \pi i}\right|=\frac{1}{2 \pi}\right\}, \quad \lim _{k \uparrow \infty} z_{\lambda, k}^{\Lambda}=0,
$$


and there exists an orthonormal sequence $\left\{\psi_{\lambda, k}^{\Lambda}\right\}_{1}^{\infty} \subset L^{2}\left(\mathbb{S}^{2}\right)$ such that for every $\psi \in L^{2}\left(\mathbb{S}^{2}\right)$,

$$
\psi=\psi_{0}+\sum_{k=1}^{\infty}\left\langle\psi_{\lambda, k}^{\Lambda}, \psi\right\rangle_{L^{2}\left(\mathbb{S}^{2}\right)} \psi_{\lambda, k}^{\Lambda}, \quad \text { where } \psi_{0} \in \operatorname{ker}\left(F_{\lambda}^{\Lambda}\right),
$$

and

$$
F_{\lambda}^{\Lambda}=\sum_{k=1}^{\infty} z_{\lambda, k}^{\Lambda} \psi_{\lambda, k}^{\Lambda} \otimes \psi_{\lambda, k}^{\Lambda}
$$

Remark 4.12. Notice that, by Remark 4.11, $\left\{\psi_{\lambda, k}^{\Lambda}\right\}_{1}^{\infty} \subset \operatorname{ker}\left(F_{\lambda}^{\Lambda}\right)^{\perp}$ and so $\operatorname{ran}\left(F_{\lambda}^{\Lambda}\right) \subseteq$ $\operatorname{ker}\left(F_{\lambda}^{\Lambda}\right)^{\perp}$.

Theorem 4.13. Let $F_{\lambda}^{\Lambda}=B C B^{*}$, where $B$ satisfies (4.11) and $C$, with $\operatorname{Im}\langle\varphi, C \varphi\rangle_{\mathrm{Y}^{*}, \mathrm{Y}} \neq 0$ for any $\varphi \in Y^{*} \backslash\{0\}$, has the decomposition $C=C_{\circ}+K$, where $C_{\circ}$ is sign-definite and $K$ is compact. Then

$$
x \in \Omega \Longleftrightarrow \sum_{k=1}^{\infty} \frac{\left|\left\langle\phi_{\lambda}^{x}, \psi_{\lambda, k}^{\Lambda}\right\rangle_{L^{2}\left(\mathbb{S}^{2}\right)}\right|^{2}}{\left|z_{\lambda, k}^{\Lambda}\right|}<+\infty
$$

where $\phi_{\lambda}^{x}$ is defined in (4.2).

Proof. Let $P_{0}: L^{2}\left(\mathbb{S}^{2}\right) \rightarrow L^{2}\left(\mathbb{S}^{2}\right)$ be the orthogonal projection such that $\operatorname{ran}\left(P_{0}\right)=L_{\perp}^{2}\left(\mathbb{S}^{2}\right):=$ $\operatorname{ker}\left(F_{\lambda}^{\Lambda}\right)^{\perp}$. Then, by Remark 4.12, $F_{\lambda}^{\Lambda}=P_{0} F_{\lambda}^{\Lambda} P_{0}$; hence $F_{\lambda}^{\Lambda}=P_{0} B C B^{*} P_{0}=\left(P_{0} B\right) C\left(P_{0} B\right)^{*}$, and so, by [18, Theorem 1.16], $\operatorname{ran}(B)=\operatorname{ran}\left(P_{0} B\right)$. Let $\widetilde{F}_{\lambda}^{\Lambda}: L_{\perp}^{2}\left(\mathbb{S}^{2}\right) \rightarrow L_{\perp}^{2}\left(\mathbb{S}^{2}\right)$ be the injective normal compact operator given by the compression of $F_{\lambda}^{\Lambda}$ to $L_{\perp}^{2}\left(\mathbb{S}^{2}\right)$. By Remark 4.11. $\left\{\psi_{\lambda, k}^{\Lambda}\right\}_{1}^{\infty}$ is an orthonormal basis in $L_{\perp}^{2}\left(\mathbb{S}^{2}\right)$ and $\widetilde{F}_{\lambda}^{\Lambda}=\sum_{k=1}^{\infty} z_{\lambda, k}^{\Lambda} \psi_{\lambda, k}^{\Lambda} \otimes \psi_{\lambda, k}^{\Lambda}$. By functional calculus for normal operators, using the factorization of $z \in \mathbb{C} \backslash\{0\}$ given by $z=|z|^{1 / 2} \operatorname{sgn}(z)|z|^{1 / 2}, \operatorname{sgn}(z):=|z|^{-1} z$, one gets

$$
\widetilde{F}_{\lambda}^{\Lambda}=\left|\widetilde{F}_{\lambda}^{\Lambda}\right|^{1 / 2} \operatorname{sgn}\left(\widetilde{F}_{\lambda}^{\Lambda}\right)\left|\widetilde{F}_{\lambda}^{\Lambda}\right|^{1 / 2} .
$$

Since $\widetilde{F}_{\lambda}^{\Lambda}=\widetilde{B} C \widetilde{B}^{*}$, where $\widetilde{B}:=P_{0} B$ (here $P_{0}$ means the surjection $P_{0}: L^{2}\left(\mathbb{S}^{2}\right) \rightarrow L_{\perp}^{2}\left(\mathbb{S}^{2}\right)$ ), by [18, Theorem 1.23], $\operatorname{ran}\left(\left|\widetilde{F}_{\lambda}^{\Lambda}\right|^{1 / 2}\right)=\operatorname{ran}(\widetilde{B})=\operatorname{ran}\left(P_{0} B\right)=\operatorname{ran}(B)$. Hence $\operatorname{ran}\left(\left|\widetilde{F}_{\lambda}^{\Lambda}\right|^{1 / 2}\right)=$ $\operatorname{ran}\left(L_{\lambda}^{\sharp} \mid \mathrm{X}_{\sharp}^{s^{*}}\right)$ and so, by Lemma 4.3 and Corollary 4.4, $x \in \Omega$ if and only if $\phi_{\lambda}^{x} \in \operatorname{ran}\left(\left|\widetilde{F}_{\lambda}^{\Lambda}\right|^{1 / 2}\right)$, equivalently if and only if $\phi_{\lambda}^{x} \in \operatorname{dom}\left(\left|\widetilde{F}_{\lambda}^{\Lambda}\right|^{-1 / 2}\right)$.

Since $\left|\widetilde{F}_{\lambda}^{\Lambda}\right|^{-1 / 2}=\sum_{k=1}^{\infty}\left|z_{\lambda, k}^{\Lambda}\right|^{-1 / 2} \psi_{\lambda, k}^{\Lambda} \otimes \psi_{\lambda, k}^{\Lambda}, \phi_{\lambda}^{x} \in \operatorname{dom}\left(\left|\widetilde{F}_{\lambda}^{\Lambda}\right|^{-1 / 2}\right)$ if and only if the series $\sum_{k=1}^{\infty}\left|z_{\lambda, k}^{\Lambda}\right|^{-1}\left|\left\langle\phi_{\lambda}^{x}, \psi_{\lambda, k}^{\Lambda}\right\rangle_{L^{2}\left(\mathbb{S}^{2}\right)}\right|^{2}$ converges.

In applications to concrete models, the following consequence of Theorems 4.9 and 4.13 turns out to be useful:

Theorem 4.14. Let

$$
F_{\lambda}^{\Lambda}=L_{\lambda}^{\sharp} \Lambda_{\lambda}^{+} L_{\lambda}^{\sharp *}, \quad \lambda \in E_{\sharp}^{-} \cap E_{\Lambda}^{-}, \quad \sharp=D, N, D N,
$$

and suppose that $\Lambda_{\lambda}^{+}=\left(M_{\lambda}^{+}\right)^{-1}$, where the bijection $M_{\lambda}^{+} \in \mathscr{B}\left(\mathbf{X}_{\sharp}^{s *}, \mathrm{X}_{\sharp}^{s}\right), s \in[0,1 / 2]$, has the decomposition $M_{\lambda}^{+}=M_{\circ}^{+}+K_{\lambda}^{+}$, with $M_{\circ}^{+}$sign-definite and $K_{\lambda}^{+}$compact. Then

$$
x \in \Omega \Longleftrightarrow \inf _{\substack{\psi \in L^{2}\left(\mathbb{S}^{2}\right) \\\left\langle\psi, \phi_{\lambda}^{x}\right\rangle_{L^{2}\left(\mathbb{S}^{2}\right)}=1}}\left|\left\langle\psi, F_{\lambda}^{\Lambda} \psi\right\rangle_{L^{2}\left(\mathbb{S}^{2}\right)}\right|>0 \Longleftrightarrow \sum_{k=1}^{\infty} \frac{\left|\left\langle\phi_{\lambda}^{x}, \psi_{\lambda, k}^{\Lambda}\right\rangle_{L^{2}\left(\mathbb{S}^{2}\right)}\right|^{2}}{\left|z_{\lambda, k}^{\Lambda}\right|}<+\infty
$$


where the sequences $\left\{z_{\lambda, k}^{\Lambda}\right\}_{1}^{\infty} \subset \mathbb{C} \backslash\{0\}$ and $\left\{\psi_{\lambda, k}^{\Lambda}\right\}_{1}^{\infty} \subset L^{2}\left(\mathbb{S}^{2}\right)$ provide the spectral resolution of $F_{\lambda}^{\Lambda}$ as in Remark 4.11 and $\phi_{\lambda}^{x}$ is defined in (4.2).

Proof. Let us consider the factorization $F_{\lambda}^{\Lambda}=\left(L_{\lambda}^{\sharp}\left(M_{\lambda}^{+}\right)^{-1}\right)\left(M_{\lambda}^{+}\right)^{*}\left(L_{\lambda}^{\sharp}\left(M_{\lambda}^{+}\right)^{-1}\right)^{*}$. Then the thesis is consequence of Lemma 4.7. Theorems 4.9 and 4.13 once one shows that

$$
\operatorname{Im}\left\langle\phi,\left(M_{\lambda}^{+}\right)^{*} \phi\right\rangle_{\mathbf{X}_{\sharp}^{s *}, X_{\sharp}^{s}} \neq 0 \text { for any } \phi \in \mathbf{X}_{\sharp}^{s *} \backslash\{0\} .
$$

Equivalently, let us prove that $\operatorname{Im}\left\langle\phi,\left(M_{\lambda}^{+}\right)^{*} \phi\right\rangle_{\mathrm{X}_{\sharp}^{s *}, \mathrm{X}_{\sharp}^{s}}=0$ implies $\phi=0$ (our reasonings below are inspired by the ones given in [18, page 51]). By the definition of $F_{\lambda}^{\Lambda}$ and since $S_{\lambda}^{\Lambda}$ is unitary, one gets

$$
F_{\lambda}^{\Lambda}-\left(F_{\lambda}^{\Lambda}\right)^{*}=-2 \pi i\left(F_{\lambda}^{\Lambda}\right)^{*} F_{\lambda}^{\Lambda} .
$$

Setting $B_{\lambda}:=L_{\lambda}^{\sharp}\left(M_{\lambda}^{+}\right)^{-1}$, this gives the identity

$$
\begin{aligned}
& \operatorname{Im}\left\langle B_{\lambda}^{*} \psi,\left(M_{\lambda}^{+}\right)^{*} B_{\lambda}^{*} \psi\right\rangle_{X_{\sharp}^{s *}, X_{\sharp}^{s}}=\operatorname{Im}\left\langle\psi, B_{\lambda}\left(M_{\lambda}^{+}\right)^{*} B_{\lambda}^{*} \psi\right\rangle_{L^{2}\left(\mathbb{S}^{2}\right)} \\
= & \frac{1}{2 i}\left\langle\psi,\left(F_{\lambda}^{\Lambda}-\left(F_{\lambda}^{\Lambda}\right)^{*}\right) \psi\right\rangle_{L^{2}\left(\mathbb{S}^{2}\right)}=-\pi\left\|F_{\lambda}^{\Lambda} \psi\right\|_{L^{2}\left(\mathbb{S}^{2}\right)}^{2} .
\end{aligned}
$$

Let $\sharp=N, D$; then by (4.5), $\operatorname{ker}\left(B_{\lambda}\right)=\operatorname{ker}\left(K_{\lambda}^{\sharp}\right)$; hence, by [18, Lemma 1.13 and Theorem 1.26(b)], one has $\operatorname{ker}\left(B_{\lambda}\right)=\{0\}$ and so $\operatorname{ran}\left(B_{\lambda}^{*}\right)$ is dense. Let $\sharp=D N$; then, by (4.8), $\operatorname{ker}\left(B_{\lambda}\right)=\operatorname{ker}\left(L^{N D}\right)=\operatorname{ker}\left(K_{\lambda}^{D} \gamma_{0} G_{\lambda}^{+}\right)$, where $G_{\lambda}^{+}(\phi \oplus \varphi):=\left(S L_{\lambda}^{+} \phi+D L_{\lambda}^{+} \varphi\right)$. Since $G_{\lambda}^{+}(\phi \oplus \varphi)$ is a radiating solution of the Helmholtz equation in $\Omega_{\mathrm{ex}}, \gamma_{0} G_{\lambda}^{+}(\phi \oplus \varphi)=0$ implies $G_{\lambda}^{+}(\phi \oplus \varphi)=$ 0 . Hence $\operatorname{ker}\left(B_{\lambda}\right)=\operatorname{ker}\left(G_{\lambda}^{+}\right)$. Since $G_{\lambda+i \epsilon}$ converges to $G_{\lambda}^{+}$in $\mathscr{B}\left(B_{2,2}^{-3 / 2}(\Gamma) \oplus H^{1 / 2}(\Gamma), L_{w}^{2}\left(\mathbb{R}^{3}\right)\right)$ (see (2.13) $)$ and there exists $c>0$ such that, for any $\epsilon>0$,

$$
\left\|G_{\lambda+i \epsilon}(\phi \oplus \varphi)\right\|_{L_{w}^{2}\left(\mathbb{R}^{3}\right)} \geq c\|\phi \oplus \varphi\|_{B_{2,2}^{-3 / 2}(\Gamma) \oplus H^{1 / 2}(\Gamma)}
$$

(see [22, proof of Lemma 3.6]), $G_{\lambda}^{+}$is injective and so $\operatorname{ran}\left(B_{\lambda}\right)$ is dense whenever $\sharp=D N$ as well.

Let $\phi \in \mathrm{X}_{\sharp}^{s *}$ be such that $\operatorname{Im}\left\langle\phi,\left(M_{\lambda}^{+}\right)^{*} \phi\right\rangle_{\mathbf{X}_{\sharp}^{s *}, \mathbf{X}_{\sharp}^{s}}=0$; let $\left\{\psi_{n}\right\}_{1}^{\infty} \subset L^{2}\left(\mathbb{S}^{2}\right)$ be a sequence such that $B_{\lambda}^{*} \psi_{n} \rightarrow \phi$. Then, by (4.12),$F_{\lambda}^{\Lambda} \psi_{n} \rightarrow 0$ and so, for any $\psi \in L^{2}\left(\mathbb{S}^{2}\right)$,

$$
\left\langle B_{\lambda}^{*} \psi,\left(M_{\lambda}^{+}\right)^{*} B_{\lambda}^{*} \psi_{n}\right\rangle_{\mathbf{X}_{\sharp}^{s *}, X_{\sharp}^{s}}=\left\langle\psi, F_{\lambda}^{\Lambda} \psi_{n}\right\rangle_{L^{2}\left(\mathbb{S}^{2}\right)} \rightarrow\left\langle B_{\lambda}^{*} \psi,\left(M_{\lambda}^{+}\right)^{*} \phi\right\rangle_{\mathbf{X}_{\sharp}^{s *}, X_{\sharp}^{s}}=0 .
$$

Therefore $\left(M_{\lambda}^{+}\right)^{*} \phi \in \operatorname{ran}\left(B_{\lambda}^{*}\right)^{\perp}=\{0\}$. Since $M_{\lambda}^{+}$is a bijection, $\left(M_{\lambda}^{+}\right)^{*}$ is injective and so $\phi=0$.

Remark 4.15. If $M_{\lambda}^{+}$in Theorem 4.14 is merely coercive, then the "inf" criterion still holds.

\subsection{Applications.}

4.1.1. Dirichlet obstacles. Let $\Delta_{\Omega_{\text {in } / \mathrm{ex}}}^{D}$ denote the self-adjoint operators in $L^{2}\left(\Omega_{\text {in/ex }}\right)$ corresponding to the Laplace operator with Dirichlet boundary conditions. One has $\Delta_{\Omega_{\mathrm{in}}}^{D} \oplus \Delta_{\Omega_{\mathrm{ex}}}^{D}=$ $\Delta_{\Lambda^{D}}$, where $\Lambda_{z}^{D}=-\left(\gamma_{0} S L_{z}\right)^{-1} \in \mathscr{B}\left(H^{1 / 2}(\Gamma), H^{-1 / 2}(\Gamma)\right), z \in \mathbb{C} \backslash(-\infty, 0]$, and Theorem 3.6 holds in this case (see [22, Section 5.2]). By first resolvent identity, $\gamma_{0} S L_{\lambda}^{+}: H^{-1 / 2}(\Gamma) \rightarrow$ $H^{1 / 2}(\Gamma)$ can be additively decomposed as $\gamma_{0} S L_{\lambda}^{+}=\gamma_{0} S L_{\mu}+(\lambda-\mu) \gamma_{0} R_{\lambda}^{0,+} S L_{\mu}$, $\mu>0$, with $\gamma_{0} S L_{\mu}$ positive (see [23, Lemma 3.2]) and $\gamma_{0} R_{\lambda}^{0,+} S L_{\mu}$ compact (see [22, Section 5.1.3]). Thus Theorem 4.14 applies to $F_{\lambda}^{\Lambda^{D}}, \lambda \in E_{D}^{-}$. 
4.1.2. Neumann obstacles. Let $\Delta_{\Omega_{\mathrm{in} / \mathrm{ex}}}^{N}$ denote the self-adjoint operators in $L^{2}\left(\Omega_{\mathrm{in} / \mathrm{ex}}\right)$ corresponding to the Laplace operator with Neumann boundary conditions. One has $\Delta_{\Omega_{\mathrm{in}}}^{N} \oplus$ $\Delta_{\Omega_{\mathrm{ex}}}^{N}=\Delta_{\Lambda^{N}}$, where $\Lambda_{z}^{N}=-\left(\gamma_{1} D L_{z}\right)^{-1} \in \mathscr{B}\left(H^{-1 / 2}(\Gamma), H^{1 / 2}(\Gamma)\right), z \in \mathbb{C} \backslash(-\infty, 0]$, and Theorem 3.6 holds in this case (see [22, Section 5.3]). By first resolvent identity, $\gamma_{1} D L_{\lambda}^{+}$: $H^{1 / 2}(\Gamma) \rightarrow H^{-1 / 2}(\Gamma)$ can be additively decomposed as $\gamma_{1} D L_{\lambda}^{+}=\gamma_{1} D L_{\mu}+(\lambda-\mu) \gamma_{1} R_{\lambda}^{0,+} D L_{\mu}$, $\mu>0$, with $-\gamma_{1} D L_{\mu}$ positive (see [23, Lemma 3.2]) and $\gamma_{1} R_{\lambda}^{0,+} D L_{\mu}$ compact (see [22, Section 5.1.3]). Thus Theorem 4.14 applies to $F_{\lambda}^{\Lambda^{N}}, \lambda \in E_{N}^{-}$.

4.1.3. Obstacles with semitransparent boundary conditions $\alpha \gamma_{0} u=\left[\gamma_{1}\right] u$. Here $\alpha$ is a realvalued function and we use the same symbol to denote the corresponding multiplication operator.

Lemma 4.16. 1) If $\alpha \in L^{6}(\Gamma)$ and $\frac{1}{\alpha} \in L^{\infty}(\Gamma)$ then $\left(\frac{1}{\alpha}+\gamma_{0} S L_{z}\right)^{-1} \in \mathscr{B}\left(L^{2}(\Gamma)\right), z \in \mathbb{C} \backslash \mathbb{R}$. 2) If both $\alpha$ and $\frac{1}{\alpha}$ belong to $L^{\infty}(\Gamma)$ and $\operatorname{sgn}(\alpha)$ is constant, then $\frac{1}{\alpha}+\gamma_{0} S L_{\lambda}^{+}, \lambda \in(-\infty, 0)$, is the sum of a sign-definite operator plus a compact one.

Proof. Since $L^{6}(\Gamma) \subseteq \mathscr{M}\left(H^{2 / 3}(\Gamma), L^{2}(\Gamma)\right)$ and $\gamma_{0} S L_{z} \in \mathscr{B}\left(H^{t-1 / 2}(\Gamma), H^{t+1 / 2}(\Gamma)\right), 0<t \leq 1 / 2$ (see [22, equation (5.27)], one has that $1+\alpha \gamma_{0} S L_{z} \in \mathscr{B}\left(L^{2}(\Gamma)\right)$ and it is injective since it is invertible (and hence injective) as a map in $H^{-1 / 3}(\Gamma)$ (use [22, Lemma 5.8]). Let us now suppose that it is not surjective from $L^{2}(\Gamma)$ onto itself, i.e. we suppose that there exists $\psi \in L^{2}(\Gamma)$ such that $\psi=\phi+\alpha \gamma_{0} S L_{z} \phi$ with $\phi \in H^{-1 / 3}(\Gamma)$, and $\phi \notin L^{2}(\Gamma)$. Hence $\alpha \gamma_{0} S L_{z} \phi \notin L^{2}(\Gamma)$, which is not possible since $S L_{z} \phi \in H^{2 / 3}(\Gamma)$ and $\alpha \in \mathscr{M}\left(H^{2 / 3}(\Gamma), L^{2}(\Gamma)\right)$. In conclusion $1+\alpha \gamma_{0} S L_{z} \in \mathscr{B}\left(L^{2}(\Gamma)\right)$ is a bounded bijection in $L^{2}(\Gamma)$ and so $\left(1+\alpha \gamma_{0} S L_{z}\right)^{-1} \in$ $\mathscr{B}\left(L^{2}(\Gamma)\right)$ by the inverse mapping theorem. Since $\alpha$ is a.e. finite, $\frac{1}{\alpha}: L^{2}(\Gamma) \rightarrow L^{2}(\Gamma)$ is a continuous bijection. Hence $\frac{1}{\alpha}+\gamma_{0} S L_{z}=\frac{1}{\alpha}\left(1+\alpha \gamma_{0} S L_{z}\right)$ is a continuous bijection and so $\left(\frac{1}{\alpha}+\gamma_{0} S L_{z}\right)^{-1} \in \mathscr{B}\left(L^{2}(\Gamma)\right)$ by the inverse mapping theorem.

Since $\gamma_{0} S L_{\lambda}^{+}$maps $L^{2}(\Gamma)$ onto $H^{1}(\Gamma)$, by the compact embedding $H^{1}(\Gamma) \hookrightarrow L^{2}(\Gamma)$, it is compact. Since $\left\langle\varphi, \frac{1}{|\alpha|} \varphi\right\rangle_{L^{2}(\Gamma)} \geq\|\alpha\|_{L^{\infty}(\Gamma)}^{-1}\|\varphi\|_{L^{2}(\Gamma)}^{2}$ and $\operatorname{sgn}(\alpha)$ is constant, $\frac{1}{\alpha}$ is signdefinite.

We consider the self-adjoint operator $\Delta_{\Lambda^{\alpha}}$, where

$$
\Lambda_{z}^{\alpha}=\left(M_{z}^{\alpha}\right)^{-1}, \quad z \in \mathbb{C} \backslash \mathbb{R}, \quad M_{z}^{\alpha}:=-\left(\frac{1}{\alpha}+\gamma_{0} S L_{z}\right) \in \mathscr{B}\left(L^{2}(\Gamma)\right) .
$$

$\Lambda_{z}^{\alpha}$ is well-defined, i.e., $M_{z}^{\alpha}$ has a bounded inverse, by Lemma 4.16. By [9, Theorem 2.19], the map $z \mapsto \Lambda_{z}^{\alpha}$ and the resolvent formula (3.5) extend to $Z_{\Lambda^{\alpha}}:=\rho\left(\Delta_{\Lambda^{\alpha}}\right) \cap \mathbb{C} \backslash(-\infty, 0]$. $\Delta_{\Lambda^{\alpha}}$ provides a self-adjoint realization of the (bounded form above) Laplacian on $\mathbb{R}^{3} \backslash \Gamma$ with the semi-transparent boundary conditions at $\Gamma$ given by $\alpha \gamma_{0} u=\left[\gamma_{1}\right] u,\left[\gamma_{0}\right] u=0$; moreover Theorem 3.6 holds in this case (see [22, Corollary 5.12]). By point 2 in Lemma 4.16, Theorem 4.14 applies to $F_{\lambda}^{\Lambda^{\alpha}}, \lambda \in E_{D}^{-}$(here $E_{\Lambda^{\alpha}}^{-}=(-\infty, 0)$ by [24, Remark 3.8]).

4.1.4. Obstacles with semitransparent boundary conditions $\gamma_{1} u=\theta\left[\gamma_{0}\right] u$. Here $\theta$ is a realvalued function and we use the same symbol to denote the corresponding multiplication operator. 
Lemma 4.17. Let $\theta \in L^{p}(\Gamma), p>2$. Then 1) $\left(\theta-\gamma_{1} D L_{z}\right)^{-1} \in \mathscr{B}\left(H^{-1 / 2}(\Gamma), H^{1 / 2}(\Gamma)\right)$, $z \in \mathbb{C} \backslash \mathbb{R}$; 2) $\theta-\gamma_{1} D L_{\lambda}^{+}, \lambda \in(-\infty, 0)$, can be decomposed as the sum of a compact operator plus a sign-definite one.

Proof. Point 1 is consequence of [22, Lemma 5.14]. Since $L^{1 / s}(\Gamma) \subseteq \mathscr{M}\left(H^{s}(\Gamma), H^{-s}(\Gamma)\right), s \in$ $[0,1]$, the map $\theta: H^{1 / 2}(\Gamma) \rightarrow H^{-1 / 2}(\Gamma)$ is compact by the compact embedding $H^{-1 / p}(\Gamma) \hookrightarrow$ $H^{-1 / 2}(\Gamma)$. The difference $\gamma_{1} D L_{\lambda}^{+}-\gamma_{1} D L_{\mu}$ is compact for any $\mu>0$ (see [22, Section 5.1.3]) and $-\gamma_{1} D L_{\mu}$ is positive (see [23, Lemma 3.2]).

We consider the self-adjoint operator $\Delta_{\Lambda^{\theta}}$,

$$
\Lambda_{z}^{\theta}=\left(M_{z}^{\theta}\right)^{-1}, \quad z \in \mathbb{C} \backslash \mathbb{R}, \quad M_{z}^{\theta}:=\theta-\gamma_{1} D L_{z} \in \mathscr{B}\left(H^{1 / 2}(\Gamma), H^{-1 / 2}(\Gamma)\right) .
$$

$\Lambda_{z}^{\theta}$ is well-defined, i.e., $M_{z}^{\theta}$ has a bounded inverse, by Lemma 4.17. By [9, Theorem 2.19], the map $z \mapsto \Lambda_{z}^{\theta}$ and the resolvent formula (3.5) extend to $Z_{\Lambda^{\theta}}:=\rho\left(\Delta_{\Lambda^{\theta}}\right) \cap \mathbb{C} \backslash(-\infty, 0]$. $\Delta_{\Lambda^{\theta}}$ provides a self-adjoint realization of the (bounded form above) Laplacian on $\mathbb{R}^{3} \backslash \Gamma$ with the semi-transparent boundary conditions at $\Gamma$ given by $\gamma_{1} u=\theta\left[\gamma_{0}\right] u,\left[\gamma_{1}\right] u=0$; moreover Theorem 3.6 holds in this case (see [22, Section 5.5]). By point 2 in Lemma 4.17, Corollary 4.7 applies to $F_{\lambda}^{\Lambda^{\theta}}, \lambda \in E_{N}^{-}$(here $E_{\Lambda^{\theta}}^{-}=(-\infty, 0)$ by [24, Remark 3.8]).

4.1.5. Obstacles with local boundary conditions.

Lemma 4.18. Let $b_{11}$ and $b_{22}$ real-valued, $b_{11}<0, b_{11} \in L^{\infty}(\Gamma), b_{11}^{-1} \in L^{\infty}(\Gamma), b_{22} \in L^{p}(\Gamma)$, $p>2, b_{12} \in \mathscr{C}^{\kappa}(\Gamma)$ for some $\kappa \in(0,1)$. Then

$$
\begin{gathered}
M_{z}^{b}: L^{2}(\Gamma) \oplus H^{1 / 2}(\Gamma) \rightarrow L^{2}(\Gamma) \oplus H^{-1 / 2}(\Gamma), \quad z \in \mathbb{C} \backslash(-\infty, 0], \\
M_{z}^{b}:=\left[\begin{array}{ll}
b_{11}+\gamma_{0} S L_{z} & b_{12}+\gamma_{0} D L_{z} \\
b_{12}^{*}+\gamma_{1} S L_{z} & b_{22}+\gamma_{1} D L_{z}
\end{array}\right]
\end{gathered}
$$

is coercive for any $z \in \mathbb{C} \backslash \mathbb{R}$.

Proof. Given $\mu>0$, let us consider the decomposition $M_{z}^{b}=M_{(1)}+M_{(2)}+M_{(3)}$, where

$$
\begin{gathered}
M_{(1)}=\left[\begin{array}{cc}
b_{11} & 0 \\
0 & \gamma_{1} D L_{\mu}
\end{array}\right] \\
M_{(2)}=\left[\begin{array}{cc}
\gamma_{0} S L_{z} & 0 \\
0 & b_{22}+\gamma_{1} D L_{z}-\gamma_{1} D L_{\mu}
\end{array}\right] \\
M_{(3)}=\left[\begin{array}{cc}
0 & b_{12}+\gamma_{0} D L_{z} \\
b_{12}^{*}+\gamma_{1} S L_{z} & 0
\end{array}\right] .
\end{gathered}
$$

By [23, Lemma 3.2],

$$
-\left\langle\varphi, \gamma_{1} D L_{\mu} \varphi\right\rangle_{H^{1 / 2}(\Gamma), H^{-1 / 2}(\Gamma)} \geq c_{\mu}\|\varphi\|_{H^{-1 / 2}(\Gamma)}^{2}, \quad c_{\mu}>0
$$

Hence, since $b_{11}<0$,

$$
-\left(\left\langle\phi, b_{11} \phi\right\rangle_{L^{2}(\Gamma)}+\left\langle\varphi, \gamma_{1} D L_{\mu} \varphi\right\rangle_{H^{1 / 2}(\Gamma), H^{-1 / 2}(\Gamma)}\right) \geq\left\|b_{11}^{-1}\right\|_{L^{\infty}(\Gamma)}^{-1}\|\phi\|_{L^{2}(\Gamma)}^{2}+c_{\mu}\|\varphi\|_{H^{-1 / 2}(\Gamma)}^{2}
$$

and $M_{(1)}$ is sign-definite. $M_{(2)}$ is compact since both its diagonal elements are compact (here one argues as in the proofs of Lemmata 4.16 and 4.17). Since $\mathscr{C}^{\kappa}(\Gamma) \subseteq \mathscr{M}\left(H^{s}(\Gamma)\right) \subseteq L^{\infty}(\Gamma)$, $0<s<\kappa$, and $\gamma_{0} D L_{z} \in \mathscr{B}\left(H^{1 / 2}(\Gamma)\right), \gamma_{1} S L_{z} \in \mathscr{B}\left(L^{2}(\Gamma)\right)$ (see, e.g., [26, Theorem 6.12 and successive remarks]), one has that $M_{(3)}$ maps $L^{2}(\Gamma) \oplus H^{1 / 2}(\Gamma)$ into $H^{s}(\Gamma) \oplus L^{2}(\Gamma)$ for 
any $s \in[0,1 / 2] \cap[0, \kappa]$; hence it is compact by the compact embeddings $H^{s}(\Gamma) \hookrightarrow L^{2}(\Gamma)$, $s>0$, and $L^{2}(\Gamma) \hookrightarrow H^{-1 / 2}(\Gamma)$. Therefore $M_{z}^{b}$ decomposes as the sum of a sign-definite operator plus a compact one. Since, by resolvent identity, $M_{z}^{b}$ satisfies (3.4), the proof is then concluded by Lemmata 3.2 and 4.7 .

By Lemma 4.18 and Remark 4.6, the operator-valued map $z \mapsto \Lambda_{z}^{b}, \Lambda_{z}^{b}:=\left(M_{z}^{b}\right)^{-1}, z \in$ $\mathbb{C} \backslash \mathbb{R}$, is well defined and, by (3.4), satisfies (3.3). Therefore, by Theorem 3.3, we can define the self-adjoint operator $\Delta_{\Lambda^{b}}$; it provides a self-adjoint realization of the Laplacian on $\mathbb{R}^{3} \backslash \Gamma$ with boundary conditions

$$
\left\{\begin{array}{l}
\gamma_{0} u=b_{11}\left[\gamma_{0}\right] u+b_{12}\left[\gamma_{1}\right] u \\
\gamma_{1} u=b_{12}^{*}\left[\gamma_{0}\right] u+b_{22}\left[\gamma_{1}\right] u
\end{array}\right.
$$

(see [23, Corollary 4.9]). By [9, Theorem 2.19], the map $z \mapsto \Lambda_{z}^{b}$ and the resolvent formula (3.5) extend to $Z_{\Lambda^{b}}:=\rho\left(\Delta_{\Lambda^{b}}\right) \cap \mathbb{C} \backslash(-\infty, 0]$. The choice

$$
b_{11}=\frac{1}{b_{\mathrm{in}}-b_{\mathrm{ex}}}, \quad b_{12}=\frac{b_{\mathrm{in}}+b_{\mathrm{ex}}}{b_{\mathrm{in}}-b_{\mathrm{ex}}}, \quad b_{22}=\frac{b_{\mathrm{in}} b_{\mathrm{ex}}}{b_{\mathrm{in}}-b_{\mathrm{ex}}},
$$

gives $\Delta_{\Lambda^{b}}=\Delta_{\Omega_{\mathrm{in}}}^{R} \oplus \Delta_{\Omega_{\mathrm{ex}}}^{R}$, where $\Delta_{\Omega_{\mathrm{in} / \mathrm{ex}}}^{R}$ denotes the Laplacian in $L^{2}\left(\Omega_{\mathrm{in} / \mathrm{ex}}\right)$ with Robin boundary conditions $\gamma_{1}^{\text {in/ex }} u_{\text {in/ex }}=b_{\text {in } / \mathrm{ex}} \gamma_{0}^{\text {in/ex }} u_{\text {in/ex }}$ (see [23, Section 5.3]). Notice that, since $\gamma_{1}^{\text {in/ex }}$ are both defined in terms of the outward normal vector, the case describing the same Robin boundary conditions at both sides of $\Gamma$ corresponds to the choice $b_{\text {in }}=b=-b_{\text {ex }}$ (thus $\left.b_{11}=\frac{1}{2 b}, b_{12}=0, b_{22}=-\frac{b}{2}\right)$.

Arguing as in [24, page 1480], one shows that $\Delta_{\Lambda^{b}}$ is bounded from above; moreover $\operatorname{ran}\left(\Lambda_{z}^{b}\right)=L^{2}(\Gamma) \oplus H^{1 / 2}(\Gamma)$ is compactly embedded in $\mathrm{K}^{*}=B_{2,2}^{-3 / 2}(\Gamma) \oplus H^{-1 / 2}(\Gamma)$. Thus Theorem 3.5 applies and the limit operator $\Lambda_{\lambda}^{b,+}$ exists for any $\lambda \in E_{\Lambda^{b}}^{-}$and $\Lambda_{\lambda}^{b,+}=\left(M_{\lambda}^{b,+}\right)^{-1}$, where

$$
M_{\lambda}^{b,+}=\left[\begin{array}{ll}
b_{11}+\gamma_{0} S L_{\lambda}^{+} & b_{12}+\gamma_{0} D L_{\lambda}^{+} \\
b_{12}^{*}+\gamma_{1} S L_{\lambda}^{+} & b_{22}+\gamma_{1} D L_{\lambda}^{+}
\end{array}\right] .
$$

Proceeding exactly in the same way as in the proof of Lemma 4.18, one shows that $M_{\lambda}^{b,+}$ is the sum of a sign-definite operator plus a compact one. Therefore Theorem 4.14 applies to $F_{\lambda}^{\Lambda^{b}}, \lambda \in E_{D N}^{-} \cap E_{\Lambda^{b}}^{-}$.

\section{Inverse Scattering For the Laplace operator With Boundary CONDitions ON NON-CLOSED LIPSCHITZ SURFACES.}

We focus now on the case of boundary conditions assigned on a relatively open subset $\Sigma$ of the boundary $\Gamma$ of the domain $\Omega$. In this framework $\Delta_{\Lambda}$ provides models of obstacles supported on the non-closed interface $\Sigma$; our aim is to determine $\Sigma$ from the knowledge of the Scattering Matrix by implementing the Factorization Method. An important difference with respect to the previous case appears: in fact the crucial coercivity hypothesis in Theorem 4.14 (by Lemma 4.7, $M_{\lambda}^{+}$there needs to be coercive) fails to hold in the spaces $\mathrm{X}_{\sharp}^{s}$, which are made of functions defined on the whole $\Gamma$ (see Notation 4.1). To avoid such a problem 
one introduces (as in [23] and 24]) projectors onto subspaces of functions supported on $\Sigma$. In the following, given $X \subset \Gamma$ closed, we use the definition

$$
H_{X}^{s}(\Gamma):=\left\{\phi \in H^{s}(\Gamma): \operatorname{supp}(\phi) \subseteq X\right\} .
$$

Given $\Sigma \subset \Gamma$ relatively open with a Lipschitz boundary, we denote by $\Pi_{\Sigma}$ the orthogonal projector in the Hilbert space $H^{s}(\Gamma),|s| \leq 1$, such that $\operatorname{ran}\left(\Pi_{\Sigma}\right)$ is the subspace orthogonal to $H_{\Sigma^{c}}^{s}(\Gamma)$.

Lemma 5.1. The orthogonal projection $\Pi_{\Sigma}$ identifies with the restriction map

$$
R_{\Sigma}: H^{s}(\Gamma) \rightarrow H^{s}(\Sigma), \quad R_{\Sigma} \phi:=\phi \mid \Sigma
$$

and its dual $\Pi_{\Sigma}^{*}$ identifies with the embedding

$$
R_{\Sigma}^{*}: H_{\bar{\Sigma}}^{-s}(\Gamma) \rightarrow H^{-s}(\Gamma), \quad R_{\Sigma}^{*} \phi=\phi .
$$

Proof. By [26, page 77], the map

$$
U_{\Sigma}: \operatorname{ran}\left(\Pi_{\Sigma}\right) \rightarrow H^{s}(\Sigma), \quad \mathrm{U}_{\Sigma}\left(\Pi_{\Sigma} \phi\right):=\left(\Pi_{\Sigma} \phi\right)|\Sigma=\phi| \Sigma
$$

is an unitary isomorphism. Therefore we can regard $H^{s}(\Sigma)$ as a closed subspace of $H^{s}(\Gamma)$. Using the decomposition $\phi=\left(1-\Pi_{\Sigma}\right) \phi \oplus U_{\Sigma}^{-1}(\phi \mid \Sigma)$, the restriction operator $R_{\Sigma} \phi:=0 \oplus$ $\mathrm{U}_{\Sigma} \Pi_{\Sigma} \phi=0 \oplus(\phi \mid \Sigma)$ is the orthogonal projection from $H^{s}(\Gamma) \simeq H_{\Sigma^{c}}^{s}(\Gamma) \oplus H^{s}(\Sigma)$ onto $H^{s}(\Sigma)$. Thus, using the identifications $\operatorname{ran}\left(\Pi_{\Sigma}\right) \simeq H^{s}(\Sigma)$ and $H_{\bar{\Sigma}}^{-s}(\Gamma) \simeq H^{s}(\Sigma)^{*}$ (see, e.g., [12, Lemma 4.3.1]), the orthogonal projection $\Pi_{\Sigma}$ identifies with $R_{\Sigma}$ and its dual $\Pi_{\Sigma}^{*}$ identifies with $R_{\Sigma}^{*}$.

Remark 5.2. Let us notice that if a bounded linear operator $M: H^{-s}(\Gamma) \rightarrow H^{s}(\Gamma)$ is coercive then $R_{\Sigma} M R_{\Sigma}^{*}: H_{\bar{\Sigma}}^{-s}(\Gamma) \rightarrow H^{s}(\Sigma)$ is coercive as well by

$$
\left|\left\langle\phi, R_{\Sigma} M R_{\Sigma}^{*} \phi\right\rangle_{H_{\bar{\Sigma}}^{-s}(\Gamma), H^{s}(\Sigma)}\right|=\left|\left\langle R_{\Sigma}^{*} \phi, M R_{\Sigma}^{*} \phi\right\rangle_{H^{-s}(\Gamma), H^{s}(\Gamma)}\right| \geq c\left\|R_{\Sigma}^{*} \phi\right\|_{H^{-s}(\Gamma)}^{2}=c\|\phi\|_{H_{\bar{\Sigma}}^{-s}(\Gamma)}^{2} .
$$

Therefore (see Remark 4.6) $\left(R_{\Sigma} M R_{\Sigma}^{*}\right)^{-1} \in \mathscr{B}\left(H^{s}(\Sigma), H_{\bar{\Sigma}}^{-s}(\Gamma)\right)$. Moreover, if $M=M_{\circ}+K$ with $M_{\circ}$ sign-definite and $K$ compact, then $R_{\Sigma} M R_{\Sigma}^{*}=R_{\Sigma} M_{\circ} R_{\Sigma}^{*}+R_{\Sigma} K R_{\Sigma}^{*}$, with $R_{\Sigma} M_{\circ} R_{\Sigma}^{*}$ sign-definite and $R_{\Sigma} K R_{\Sigma}^{*}$ compact. Analogously, if $\operatorname{Im}\langle\phi, M \phi\rangle_{H^{-s}(\Gamma), H^{s}(\Gamma)}=0$ implies $\phi=0$, then $\operatorname{Im}\left\langle\phi, R_{\Sigma} M R_{\Sigma}^{*} \phi\right\rangle_{H_{\bar{\Sigma}}^{-s}(\Gamma), H^{s}(\Sigma)}=0$ implies $R_{\Sigma}^{*} \phi=0$ and hence $\phi=0$.

The same considerations apply to $M: H^{-s}(\Gamma) \oplus H^{-t}(\Gamma) \rightarrow H^{s}(\Gamma) \oplus H^{t}(\Gamma)$ and $\left(R_{\Sigma} \oplus\right.$ $\left.R_{\Sigma}\right) M\left(R_{\Sigma}^{*} \oplus R_{\Sigma}^{*}\right): H_{\bar{\Sigma}}^{-s}(\Gamma) \oplus H_{\bar{\Sigma}}^{-t}(\Gamma) \rightarrow H^{s}(\Sigma) \oplus H^{t}(\Sigma)$.

In the following $\Gamma_{\circ}$ is the Lipschitz boundary of an open bounded set $\Omega_{\circ} \subset \mathbb{R}^{3}$ and $\Sigma_{\circ} \subset \Gamma_{\circ}$ is relatively open with Lipschitz boundary.

Lemma 5.3. Let $\Sigma \subset \Gamma$ and $\Sigma_{\circ} \subset \Gamma_{\circ}$ such that $\mathbb{R}^{3} \backslash\left(\Sigma_{\circ} \cup \Sigma\right)$ is connected. Then

$$
\Sigma_{\circ} \subset \Sigma \Longleftrightarrow \phi_{\lambda}^{\Sigma_{\circ}} \in \operatorname{ran}\left(L_{\lambda}^{\sharp} \mid H_{\bar{\Sigma}}^{s-s_{\sharp}}(\Gamma)\right), \quad \sharp=D, N,
$$

where

$$
\phi_{\lambda}^{\Sigma_{\circ}}(\xi):=\int_{\Sigma_{\circ}} \phi_{\lambda}^{x}(\xi) d \sigma_{\Gamma_{\circ}}(x) \equiv \int_{\Sigma_{\circ}} e^{i|\lambda|^{1 / 2} \xi \cdot x} d \sigma_{\Gamma_{\circ}}(x)
$$


Proof. Let $\widetilde{u}_{\lambda, \phi}^{\sharp}$ be the radiating (i.e satisfying the Sommerfeld radiating condition) solution in $\mathbb{R}^{3} \backslash \bar{\Sigma}$ of Helmholtz equation $(-\Delta+\lambda) \widetilde{u}_{\lambda, \phi}^{\sharp}=0$ with either Dirichlet (whenever $\sharp=D$ ) or Neumann (whenever $\sharp=N$ ) boundary condition $\phi \in H^{s_{\sharp}}(\Sigma)$. Such a solution exists and is unique in

$$
\begin{aligned}
& H_{\Delta, l o c}^{1}\left(\mathbb{R}^{3} \backslash \bar{\Sigma}\right):= \\
& \left\{u \in \mathscr{D}^{\prime}\left(\mathbb{R}^{3} \backslash \bar{\Sigma}\right): u_{B} \in H^{1}\left(B \cap \mathbb{R}^{3} \backslash \bar{\Sigma}\right), \Delta u_{B} \in L^{2}\left(B \cap \mathbb{R}^{3} \backslash \bar{\Sigma}\right) \text { for any open ball } B \supset \bar{\Omega}\right\},
\end{aligned}
$$

where $u_{B}:=u \mid B \cap \mathbb{R}^{3} \backslash \bar{\Sigma}$ (see [1, Theorems 3.1 and 3.3], see also [2, Section 12.8] and, for the case with smooth boundaries, [32]). Then (see, e.g., [26, Exercise 9.4(iv)]) there exists a unique $\widetilde{u}_{\lambda, \phi}^{\sharp, \infty} \in \mathscr{C}^{\infty}\left(\mathbb{S}^{2}\right)$ such that

$$
\widetilde{u}_{\lambda, \phi}^{\sharp}(x)=\frac{e^{i|\lambda|^{1 / 2}\|x\|}}{4 \pi\|x\|} \widetilde{u}_{\lambda, \phi}^{\sharp, \infty}(\hat{x})+O\left(\|x\|^{-2}\right) \quad \text { as }\|x\| \rightarrow+\infty \text {, uniformly in } \hat{x}:=x /\|x\| .
$$

This defines the data-to-pattern operator

$$
\widetilde{K}_{\lambda}^{\sharp}: H^{s_{\sharp}}(\Sigma) \rightarrow L^{2}\left(\mathbb{S}^{2}\right), \quad \widetilde{K}_{\lambda}^{\sharp} \phi:=\widetilde{u}_{\lambda, \phi}^{\sharp, \infty} .
$$

Introducing the Herglotz operators

$$
\widetilde{H}_{\lambda}^{\sharp}: L^{2}\left(\mathbb{S}^{2}\right) \rightarrow H^{s_{\sharp}}(\Sigma), \quad \widetilde{H}_{\lambda}^{\sharp}:=R_{\Sigma} H_{\lambda}^{\sharp},
$$

where $H_{\lambda}^{\sharp}$ is defined in (4.3) , one has, for any $\phi \in H_{\bar{\Sigma}}^{-s_{\sharp}^{\sharp}}(\Gamma)$ and $f \in L^{2}\left(\mathbb{S}^{2}\right)$,

$$
\left\langle L_{\lambda}^{\sharp} \phi, f\right\rangle_{L^{2}\left(\mathbb{S}^{2}\right)}=\frac{1}{2^{1 / 2}} \frac{|\lambda|^{1 / 4}}{(2 \pi)^{3 / 2}}\left\langle\phi, \widetilde{H}_{\lambda}^{\sharp} f\right\rangle_{H_{\bar{\Sigma}}^{-s_{\sharp}}(\Gamma), H^{s_{\sharp}}(\Sigma)}=\frac{1}{2^{1 / 2}} \frac{|\lambda|^{1 / 4}}{(2 \pi)^{3 / 2}}\left\langle\left(\widetilde{H}_{\lambda}^{\sharp}\right)^{*} \phi, f\right\rangle_{L^{2}\left(\mathbb{S}^{2}\right)} .
$$

Proceeding as in [18, proofs of Theorems 1.15 and 1.26] leading to (4.4), one gets

$$
\left(\widetilde{H}_{\lambda}^{D}\right)^{*}=\widetilde{K}_{\lambda}^{D} R_{\Sigma} \gamma_{0} S L_{\lambda}^{+} R_{\Sigma}^{*}, \quad\left(\widetilde{H}_{\lambda}^{N}\right)^{*}=\widetilde{K}_{\lambda}^{N} R_{\Sigma} \gamma_{1} D L_{\lambda}^{+} R_{\Sigma}^{*},
$$

and so

$$
L_{\lambda}^{D}=\frac{1}{2^{1 / 2}} \frac{|\lambda|^{1 / 4}}{(2 \pi)^{3 / 2}} \widetilde{K}_{\lambda}^{D} R_{\Sigma} \gamma_{0} S L_{\lambda}^{+} R_{\Sigma}^{*}, \quad L_{\lambda}^{N}=\frac{1}{2^{1 / 2}} \frac{|\lambda|^{1 / 4}}{(2 \pi)^{3 / 2}} \widetilde{K}_{\lambda}^{N} R_{\Sigma} \gamma_{1} D L_{\lambda}^{+} R_{\Sigma}^{*} .
$$

By the mapping properties of $S L_{\lambda}^{+}$and $D L_{\lambda}^{+}$and by Remark [5.2, one has $R_{\Sigma} \gamma_{0} S L_{\lambda}^{+} R_{\Sigma}^{*} \in$ $\mathscr{B}\left(H_{\bar{\Sigma}}^{s-1 / 2}(\Gamma), H^{s+1 / 2}(\Sigma)\right)$ and $R_{\Sigma} \gamma_{1} D L_{\lambda}^{+} R_{\Sigma}^{*} \in \mathscr{B}\left(H_{\bar{\Sigma}}^{s+1 / 2}(\Gamma), H^{s-1 / 2}(\Sigma)\right), s \in[0,1 / 2]$. These maps are bijections (by (5.4), (5.5) in next Subsections 5.1.1 and 5.1.2 and by the regularity results in [10, Theorem 3]; see also [32] for the case of smooth boundaries), and so

$$
\operatorname{ran}\left(L_{\lambda}^{\sharp} \mid H_{\bar{\Sigma}}^{s-s_{\sharp}}(\Gamma)\right)=\operatorname{ran}\left(\widetilde{K}_{\lambda}^{\sharp} \mid H^{s+s_{\sharp}}(\Sigma)\right) .
$$

Therefore to conclude the proof we need to show that

$$
\Sigma_{\circ} \subset \Sigma \Longleftrightarrow \phi_{\lambda}^{\Sigma_{\circ}} \in \operatorname{ran}\left(\widetilde{K}_{\lambda}^{\sharp} \mid H^{s+s_{\sharp}}(\Sigma)\right) .
$$

Here we follows the same kind of reasonings as in [19, Section 3.2]. Assume that $\Sigma_{\circ} \subset \Sigma$; let $u_{\lambda}^{\sharp, \Sigma_{\circ}}$ be defined according to

$$
u_{\lambda}^{D, \Sigma_{\circ}}:=S L_{\lambda}^{+} 1_{\Sigma_{\circ}}, \quad u_{\lambda}^{N, \Sigma_{\circ}}:=D L_{\lambda}^{+} 1_{\Sigma_{\circ}} .
$$

It solves the Helmoltz equation $(-\Delta+\lambda) u_{\lambda}^{\sharp \Sigma_{\circ}}=0$ in $\mathbb{R}^{3} \backslash \overline{\Sigma_{\circ}}$ and hence in $\mathbb{R}^{3} \backslash \bar{\Sigma}$ as well. Let $\phi_{\Sigma_{\circ}}^{D}:=R_{\Sigma} \gamma_{0} u_{\lambda}^{D, \Sigma_{\circ}} \in H^{1 / 2}(\Sigma), \phi_{\Sigma_{\circ}}^{N}:=R_{\Sigma} \gamma_{1} u_{\lambda}^{N, \Sigma_{\circ}} \in H^{-1 / 2}(\Sigma)$. Then $\widetilde{K}_{\lambda}^{\sharp} \phi_{\Sigma_{\circ}}^{\sharp}=\phi_{\lambda}^{\Sigma_{\circ}}$. 
Suppose now that $\Sigma_{\circ} \cap \Sigma^{c} \neq \emptyset$. Let $B \subset \mathbb{R}^{3}$ be an open ball such that $\bar{B} \cap \bar{\Sigma}=\emptyset$, $B \cap \Sigma_{\circ} \neq \emptyset$. Assume that $\phi_{\lambda}^{\Sigma_{\circ}}=\widetilde{K}_{\lambda}^{\sharp} \phi_{\sharp}$ for some $\phi_{\sharp} \in H^{s+s_{\sharp}}(\Sigma)$ and consider the corresponding

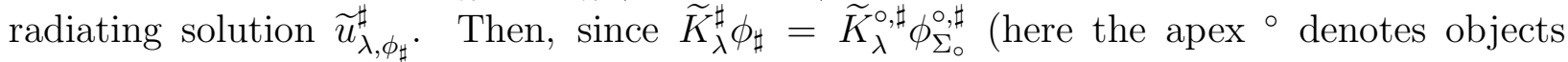
defined by using the surface $\Gamma_{\circ}$ ), one has, by Rellich's Lemma and unique continuation, $\widetilde{u}_{\lambda, \phi_{\sharp}}^{\sharp}\left|\mathbb{R}^{3} \backslash\left(\Sigma_{\circ} \cup \Sigma\right)=u_{\lambda}^{\circ, \sharp, \Sigma_{\circ}}\right| \mathbb{R}^{3} \backslash\left(\Sigma_{\circ} \cup \Sigma\right)$. By elliptic regularity, $(-\Delta+\lambda) \widetilde{u}_{\lambda, \phi}^{\sharp} \mid B=0$ implies $\widetilde{u}_{\lambda, \phi}^{\sharp} \mid B \in H^{2}(B)$; this leads to a contradiction, since $u_{\lambda}^{\circ, \sharp, \Sigma_{\circ}} \mid B \notin H^{2}(B)$.

By the same kind of proof provided for Corollary 4.4, one gets the following:

Corollary 5.4. Let $\Sigma \subset \Gamma$ and $\Sigma_{\circ} \subset \Gamma_{\circ}$ such that $\mathbb{R}^{3} \backslash\left(\Sigma_{\circ} \cup \Sigma\right)$ is connected. Then

$$
\Sigma_{\circ} \subset \Sigma \Longleftrightarrow \phi_{\lambda}^{\Sigma_{\circ}} \in \operatorname{ran}\left(L_{\lambda}^{D N} \mid H_{\bar{\Sigma}}^{s-1 / 2}(\Gamma) \oplus H_{\bar{\Sigma}}^{t+1 / 2}(\Gamma)\right), \quad s, t \in[0,1 / 2] .
$$

Notation 5.5. We introduce the spaces

$$
\widetilde{\mathrm{X}}_{D}^{s}:=H^{1 / 2-s}(\Sigma), \quad \widetilde{\mathrm{X}}_{N}^{s}:=H^{-1 / 2-s}(\Sigma), \quad \widetilde{\mathrm{X}}_{D N}^{s}:=H^{1 / 2-s}(\Sigma) \oplus H^{-s}(\Sigma), \quad 0 \leq s \leq 1 / 2,
$$

so that

$$
\left(\widetilde{\mathrm{X}}_{D}^{s}\right)^{*}:=H_{\bar{\Sigma}}^{s-1 / 2}(\Gamma), \quad\left(\widetilde{\mathrm{X}}_{N}^{s}\right)^{*}:=H_{\bar{\Sigma}}^{s+1 / 2}(\Gamma), \quad\left(\widetilde{\mathrm{X}}_{D N}^{s}\right)^{*}:=H_{\bar{\Sigma}}^{s-1 / 2}(\Gamma) \oplus H_{\bar{\Sigma}}^{s}(\Gamma), \quad 0 \leq s \leq 1 / 2 .
$$

The following results is the analogue for screens of Theorem 4.14:

Theorem 5.6. Let

$$
F_{\lambda}^{\Lambda}=L_{\lambda}^{\sharp} \Lambda_{\lambda}^{+} L_{\lambda}^{\sharp *}, \quad \lambda \in E_{\Lambda}^{-}, \quad \sharp=D, N, D N,
$$

and suppose that $\Lambda_{\lambda}^{+}=R_{\Sigma}^{*}\left(M_{\lambda}^{+, \Sigma}\right)^{-1} R_{\Sigma}$, where the bijection $M_{\lambda}^{+, \Sigma} \in \mathscr{B}\left(\left(\widetilde{\mathrm{X}}_{\sharp}^{s}\right)^{*}, \widetilde{\mathrm{X}}_{\sharp}^{s}\right), s \in$ $[0,1 / 2]$, has the decomposition $M_{\lambda}^{+, \Sigma}=M_{\circ}^{+, \Sigma}+K_{\lambda}^{+, \Sigma}$, where $M_{\circ}^{+, \Sigma}$ is sign-definite and $K_{\lambda}^{+, \Sigma}$ is compact. Let $\Sigma_{\circ} \subset \Gamma_{\circ}$ such that $\mathbb{R}^{3} \backslash\left(\Sigma_{\circ} \cup \Sigma\right)$ is connected; then

$$
\Sigma_{\circ} \subset \Sigma \Longleftrightarrow \inf _{\substack{\psi \in L^{2}\left(\mathbb{S}^{2}\right) \\\left\langle\psi, \phi_{\lambda}^{\Sigma_{0}}\right\rangle_{L^{2}\left(\mathbb{S}^{2}\right)}=1}}\left|\left\langle\psi, F_{\lambda}^{\Lambda} \psi\right\rangle_{L^{2}\left(\mathbb{S}^{2}\right)}\right|>0 \Longleftrightarrow \sum_{k=1}^{\infty} \frac{\left|\left\langle\phi_{\lambda}^{\Sigma_{\circ}}, \psi_{\lambda, k}^{\Lambda}\right\rangle_{L^{2}\left(\mathbb{S}^{2}\right)}\right|^{2}}{\left|z_{\lambda, k}^{\Lambda}\right|}+\infty,
$$

where the sequences $\left\{z_{\lambda, k}^{\Lambda}\right\}_{1}^{\infty} \subset \mathbb{C} \backslash\{0\}$ and $\left\{\psi_{\lambda, k}^{\Lambda}\right\}_{1}^{\infty} \subset L^{2}\left(\mathbb{S}^{2}\right)$ provide the spectral resolution of $F_{\lambda}^{\Lambda}$ as in Remark 4.11 and $\phi_{\lambda}^{\Sigma_{\circ}}$ is defined in (5.1).

Proof. We use the factorization $F_{\lambda}^{\Lambda}=\left(L_{\lambda}^{\sharp} R_{\Sigma}^{*}\left(M_{\lambda}^{+, \Sigma}\right)^{-1}\right)\left(M_{\lambda}^{+, \Sigma}\right)^{*}\left(L_{\lambda}^{\sharp} R_{\Sigma}^{*}\left(M_{\lambda}^{+, \Sigma}\right)^{-1}\right)^{*}$. By proceeding as in the proof of Theorem 4.14 (where now $B_{\lambda}=L_{\lambda}^{\sharp} R_{\Sigma}^{*}\left(M_{\lambda}^{+, \Sigma}\right)^{-1}$ ), one gets $\operatorname{Im}\left\langle\phi, M_{\lambda}^{+, \Sigma} \phi\right\rangle_{\left(\widetilde{X}_{\sharp}^{s}\right)^{*}, \widetilde{X}_{\sharp}^{s}} \neq 0$ for any $\phi \neq 0$. Since $M_{\lambda}^{+, \Sigma}$ is a bijection, one has $\operatorname{ran}\left(L_{\lambda}^{\sharp} R_{\Sigma}^{*}\left(M_{\lambda}^{+, \Sigma}\right)^{-1}\right)=$ $\operatorname{ran}\left(L_{\lambda}^{\sharp} \mid\left(\widetilde{X}_{\sharp}^{s}\right)^{*}\right)$. Hence, by Lemma 5.3, Corollary [5.4 and by [18, Theorem 1.16],

$$
\Sigma_{\circ} \subset \Sigma \Longleftrightarrow \inf _{\substack{\psi \in L^{2}\left(\mathbb{S}^{2}\right) \\\left\langle\psi, \phi_{\lambda}^{\Sigma_{0}}\right\rangle_{L^{2}\left(\mathbb{S}^{2}\right)}=1}}\left|\left\langle\psi, F_{\lambda}^{\Lambda} \psi\right\rangle_{L^{2}\left(\mathbb{S}^{2}\right)}\right|>0
$$

By proceeding as in the proof of Theorem 4.13, $\operatorname{ran}\left(L_{\lambda}^{\sharp} R_{\Sigma}^{*}\left(M_{\lambda}^{+, \Sigma}\right)^{-1}\right)=\operatorname{ran}\left(\left|\widetilde{F}_{\lambda}^{\Lambda}\right|^{1 / 2}\right)$ and then one concludes by the same arguments.

Remark 5.7. If $M_{\lambda}^{+, \Sigma}$ in Theoren 5.6 is merely coercive, then the "inf" criterion still holds. 
5.1. Applications. Here we apply Theorem 5.6 the analogue of models in the examples considered in Section 4.1 where now the boundary conditions holds only on $\Sigma$. Before considering the specific examples, let us explain our strategy.

At first, notice that all the examples in Section 4.1 consider self-adjoint operators $\Delta_{\Lambda}$ with $\Lambda_{z}=M_{z}^{-1}$, where the map $z \mapsto M_{z}$ satisfies (3.4) (see Remark 3.1). Hence, by Lemma 3.2. $\operatorname{Im}\left\langle\phi, M_{z} \phi\right\rangle_{\mathrm{X}_{\sharp}^{s *}, \mathrm{X}_{\sharp}^{s}} \neq 0$ for any $\phi \neq 0$. Furthermore, all such $M_{z}$ 's have a decomposition $M_{z}=M_{\circ}+K_{z}$ with $M_{\circ}$ sign-definite, $K_{z}$ compact; this can be checked by proceeding as in the proof of Lemma 4.18 using identities (2.14). Then, by Lemma 4.7, $M_{z}$ is coercive. Now, the dual couple of projectors $R_{\Sigma}, R_{\Sigma}^{*}$ in Lemma 5.1 come into play: by Remark 5.2, these properties of $M_{z}$ transfer to $M_{z}^{\Sigma}:=R_{\Sigma} M_{z} R_{\Sigma}^{*}$ (here and in the following lines, $R_{\Sigma}$ has to be replaced by $R_{\Sigma} \oplus R_{\Sigma}$ when one considers example in Subsection 4.1.5), and so, in particular, $M_{z}^{\Sigma}$ is coercive and $\left(R_{\Sigma} M_{z} R_{\Sigma}^{*}\right)^{-1} \in \mathscr{B}\left(\widetilde{\mathrm{X}}_{\sharp}^{s},\left(\widetilde{\mathrm{X}}_{\sharp}^{s}\right)^{*}\right)$. Then, setting

$$
\widetilde{\Lambda}_{z}:=R_{\Sigma}^{*}\left(R_{\Sigma} M_{z} R_{\Sigma}^{*}\right)^{-1} R_{\Sigma}
$$

it is immediate to check that $z \mapsto \widetilde{\Lambda}_{z} \in \mathscr{B}\left(\mathrm{X}_{\sharp}^{s}, \mathrm{X}_{\sharp}^{s *}\right)$ satisfies (3.3), and so, by Theorem 3.3. it defines a self-adjoint operator $\Delta_{\widetilde{\Lambda}}$. Such an operator describes the model corresponding to the same kind of boundary conditions associated to $\Delta_{\Lambda}$, now assigned only on $\Sigma$ (see [23, Section 6], [24, Section 7]). Since the limit operator $M_{\lambda}^{+}$exists (use (2.13)) and, by Theorem [3.6, the limit $\widetilde{\Lambda}_{\lambda}^{+}$exists as well, one gets $\widetilde{\Lambda}_{\lambda}^{+}=R_{\Sigma}^{*}\left(R_{\Sigma} M_{\lambda}^{+} R_{\Sigma}^{*}\right)^{-1} R_{\Sigma}$. Now, since all $M_{\lambda}^{+}$appearing in the examples in Section 4.1 decompose as the sum of a sign-definite operator plus a compact one, by Remark 5.2 the same is true for $R_{\Sigma} M_{\lambda}^{+} R_{\Sigma}^{*}$. In conclusion, the assumptions in Theorem 5.6 hold for any $\widetilde{\Lambda}$ defined as in (5.3) where $M_{z}$ is any of the operators given in the examples in Section 4.1, hence the reconstruction formula (5.2) applies to $F_{\lambda}^{\widetilde{\Lambda}}$. In what follows this scheme is implemented case by case.

5.1.1. Dirichlet screens. One considers $\Delta_{\widetilde{\Lambda}^{D}}$ with

$$
\widetilde{\Lambda}_{z}^{D}=-R_{\Sigma}^{*}\left(R_{\Sigma} \gamma_{0} S L_{z} R_{\Sigma}^{*}\right)^{-1} R_{\Sigma} \in \mathscr{B}\left(H^{1 / 2}(\Gamma), H^{-1 / 2}(\Gamma)\right), \quad z \in \mathbb{C} \backslash \mathbb{R} .
$$

$\Delta_{\widetilde{\Lambda}^{D}}$ is a (bounded from above) self-adjoint representation of the Laplacian on $\mathbb{R}^{3} \backslash \bar{\Sigma}$ with homogeneous Dirichlet boundary conditions at $\Sigma$ (see [24, Example 7.1]). By [9, Theorem 2.19], the map $z \mapsto \widetilde{\Lambda}_{z}^{D}$ and the corresponding resolvent formula (3.5) extends to $Z_{\widetilde{\Lambda}^{D}}:=$ $\rho\left(\Delta_{\widetilde{\Lambda}^{D}}\right) \cap \mathbb{C} \backslash(-\infty, 0]=\mathbb{C} \backslash(-\infty, 0]$. By [24, Theorem 3.7], $\sigma_{\mathrm{p}}^{-}\left(\Delta_{\widetilde{\Lambda}^{D}}\right)$ is empty and so, by Theorem 3.6.

$$
\forall \lambda \in(-\infty, 0), \quad\left(R_{\Sigma} \gamma_{0} S L_{\lambda}^{+} R_{\Sigma}^{*}\right)^{-1} \in \mathscr{B}\left(H^{1 / 2}(\Sigma), H_{\bar{\Sigma}}^{-1 / 2}(\Gamma)\right) .
$$

Therefore Theorem 5.6 applies to $F_{\lambda}^{\widetilde{\Lambda}^{D}}, \lambda \in(-\infty, 0)$.

\subsubsection{Neumann screens. One considers $\Delta_{\widetilde{\Lambda}^{N}}$ with}

$$
\widetilde{\Lambda}_{z}^{N}=-R_{\Sigma}^{*}\left(R_{\Sigma} \gamma_{1} D L_{z} R_{\Sigma}^{*}\right)^{-1} R_{\Sigma} \in \mathscr{B}\left(H^{-1 / 2}(\Gamma), H^{1 / 2}(\Gamma)\right), \quad z \in \mathbb{C} \backslash \mathbb{R} .
$$

$\Delta_{\widetilde{\Lambda}^{N}}$ is a (bounded from above) self-adjoint representation of the Laplacian on $\mathbb{R}^{3} \backslash \bar{\Sigma}$ with homogeneous Neumann boundary conditions at $\Sigma$ (see [24, Example 7.2]). By [9, Theorem 2.19], the map $z \mapsto \widetilde{\Lambda}_{z}^{N}$ and the corresponding resolvent formula (3.5) extends to $Z_{\widetilde{\Lambda}^{N}}:=$ 
$\rho\left(\Delta_{\widetilde{\Lambda}^{N}}\right) \cap \mathbb{C} \backslash(-\infty, 0]=\mathbb{C} \backslash(-\infty, 0]$. By [24, Theorem 3.7], $\sigma_{\mathrm{p}}^{-}\left(\Delta_{\widetilde{\Lambda}^{N}}\right)$ is empty and so, by Theorem 3.6.

$$
\forall \lambda \in(-\infty, 0), \quad\left(R_{\Sigma} \gamma_{1} D L_{\lambda}^{+} R_{\Sigma}^{*}\right)^{-1} \in \mathscr{B}\left(H^{-1 / 2}(\Sigma), H_{\bar{\Sigma}}^{1 / 2}(\Gamma)\right) .
$$

Therefore Theorem 5.6 applies to $F_{\lambda}^{\widetilde{\Lambda}^{N}}, \lambda \in(-\infty, 0)$.

5.1.3. Screens with semitransparent boundary conditions $\alpha_{\Sigma} \gamma_{0} u=\left[\gamma_{1}\right] u$. Let $\alpha \in L^{\infty}(\Gamma)$ real-valued such that $\operatorname{sgn}(\alpha)$ is constant and $\frac{1}{\alpha} \in L^{\infty}(\Gamma)$; let us define $\alpha_{\Sigma}:=\alpha \mid \Sigma$ and $\alpha_{\Sigma}^{-1} \in \mathscr{B}\left(L_{\bar{\Sigma}}^{2}(\Gamma), L^{2}(\Sigma)\right)$ by $\alpha_{\Sigma}^{-1} \phi:=\left(\alpha^{-1} \phi\right) \mid \Sigma$. Since $-\left(\alpha_{\Sigma}^{-1}+R_{\Sigma} \gamma_{0} S L_{z} R_{\Sigma}^{*}\right)=R_{\Sigma} M_{z}^{\alpha} R_{\Sigma}^{*}$, where $M_{z}^{\alpha}$ is defined in (4.13), one considers $\Delta_{\widetilde{\Lambda}^{\alpha}}$, where

$$
\widetilde{\Lambda}_{z}^{\alpha}=R_{\Sigma}^{*}\left(R_{\Sigma} M_{z}^{\alpha} R_{\Sigma}^{*}\right)^{-1} R_{\Sigma} \in \mathscr{B}\left(L^{2}(\Gamma)\right), \quad z \in \mathbb{C} \backslash(-\infty, 0] .
$$

$\Delta_{\widetilde{\Lambda}^{\alpha}}$ is a self-adjoint representation of the (bounded from above) Laplacian on $\mathbb{R}^{3} \backslash \bar{\Sigma}$ with boundary conditions at $\Sigma$ given by $\alpha_{\Sigma} R_{\Sigma} \gamma_{0} u=R_{\Sigma}\left[\gamma_{1}\right] u, R_{\Sigma}\left[\gamma_{0}\right] u=0$ (see [24, Example 7.4]). By [9, Theorem 2.19], the map $z \mapsto \widetilde{\Lambda}_{z}^{\alpha}$ and the resolvent formula (3.5) extend to $Z_{\widetilde{\Lambda}^{\alpha}}:=\rho\left(\Delta_{\widetilde{\Lambda}^{\alpha}}\right) \cap \mathbb{C} \backslash(-\infty, 0]$. By [24, Theorem 3.7], $\sigma_{\mathrm{p}}^{-}\left(\Delta_{\widetilde{\Lambda}^{\alpha}}\right)$ is empty and so, by Theorem 3.6 ,

$$
\forall \lambda \in(-\infty, 0), \quad\left(\alpha_{\Sigma}^{-1}+R_{\Sigma} \gamma_{0} S L_{\lambda}^{+} R_{\Sigma}^{*}\right)^{-1} \in \mathscr{B}\left(L^{2}(\Sigma), L_{\bar{\Sigma}}^{2}(\Gamma)\right) .
$$

Therefore Theorem 5.6 applies to $F_{\lambda}^{\widetilde{\Lambda}^{\alpha}}, \lambda \in(-\infty, 0)$.

5.1.4. Screens with semitransparent boundary conditions $\theta_{\Sigma} \gamma_{1} u=\left[\gamma_{0}\right] u$. Let $\theta \in L^{p}(\Gamma)$, $p>2$; set $\theta_{\Sigma}:=\theta \mid \Sigma$ and define the corresponding operator $\theta_{\Sigma} \in \mathscr{B}\left(H_{\bar{\Sigma}}^{1 / 2}(\Gamma), H^{-1 / 2}(\Sigma)\right)$ by $\theta_{\Sigma} \varphi:=(\theta \varphi) \mid \Sigma$. Since $\left(\theta_{\Sigma}-R_{\Sigma} \gamma_{1} D L_{z} R_{\Sigma}^{*}\right)=R_{\Sigma} M_{z}^{\theta} R_{\Sigma}^{*}$, where $M_{z}^{\theta}$ is defined in (4.14), one considers $\Delta_{\widetilde{\Lambda}^{\theta}}$, where

$$
\widetilde{\Lambda}_{z}^{\theta}=R_{\Sigma}^{*}\left(R_{\Sigma} M_{z}^{\theta} R_{\Sigma}^{*}\right)^{-1} R_{\Sigma} \in \mathscr{B}\left(H^{-1 / 2}(\Gamma), H^{1 / 2}(\Gamma)\right), \quad z \in \mathbb{C} \backslash \mathbb{R} .
$$

$\Delta_{\widetilde{\Lambda}^{\theta}}$ is a self-adjoint representation of the (bounded from above) Laplacian on $\mathbb{R}^{3} \backslash \bar{\Sigma}$ with boundary conditions at $\Sigma$ given by $\theta_{\Sigma} R_{\Sigma} \gamma_{1} u=R_{\Sigma}\left[\gamma_{0}\right] u, R_{\Sigma}\left[\gamma_{1}\right] u=0$ (see [24, Example 7.5]). By [9, Theorem 2.19], the map $z \mapsto \widetilde{\Lambda}_{z}^{\theta}$ and the resolvent formula (3.5) extend to $Z_{\widetilde{\Lambda}^{\theta}}:=\rho\left(\Delta_{\widetilde{\Lambda}^{\theta}}\right) \cap \mathbb{C} \backslash(-\infty, 0]$. By [24, Theorem 3.7], $\sigma_{\mathrm{p}}^{-}\left(\Delta_{\widetilde{\Lambda}^{\theta}}\right)$ is empty and so, by Theorem 3.6 ,

$$
\forall \lambda \in(-\infty, 0), \quad\left(\theta_{\Sigma}+R_{\Sigma} \gamma_{1} D L_{\lambda}^{+} R_{\Sigma}^{*}\right)^{-1} \in \mathscr{B}\left(H^{-1 / 2}(\Sigma), H_{\bar{\Sigma}}^{1 / 2}(\Gamma)\right) .
$$

Therefore Theorem 5.6 applies to $F_{\lambda}^{\widetilde{\Lambda}^{\theta}}, \lambda \in(-\infty, 0)$.

5.1.5. Screens with local boundary conditions. Let $b_{11} \in L^{\infty}(\Gamma), b_{11}^{-1} \in L^{\infty}(\Gamma), b_{22} \in L^{p}(\Gamma)$, $p>2, b_{12} \in \mathscr{C}^{\kappa}(\Gamma), 0<\kappa<1$, with $b_{11}$ and $b_{22}$ real-valued, $b_{11}<0$. Set $b_{i j}^{\Sigma}:=b_{i j} \mid \Sigma$ and define the corresponding multiplication operator by $b_{i j}^{\Sigma} \phi:=\left(b_{i j} \phi\right) \mid \Sigma$, where $\operatorname{supp}(\phi) \subseteq \bar{\Sigma}$. Since

$$
\left[\begin{array}{cc}
b_{11}^{\Sigma}+R_{\Sigma} \gamma_{0} S L_{z} R_{\Sigma}^{*} & b_{12}^{\Sigma}+R_{\Sigma} \gamma_{0} D L_{z} R_{\Sigma}^{*} \\
\left(b_{12}^{\Sigma}\right)^{*}+R_{\Sigma} \gamma_{1} S L_{z} R_{\Sigma}^{*} & b_{22}^{\Sigma}+R_{\Sigma} \gamma_{1} D L_{z} R_{\Sigma}^{*}
\end{array}\right]=\left(R_{\Sigma} \oplus R_{\Sigma}\right) M_{z}^{b}\left(R_{\Sigma}^{*} \oplus R_{\Sigma}^{*}\right),
$$

where $M_{z}^{b}$ is defined in Lemma (4.18), one considers $\Delta_{\widetilde{\Lambda}^{b}}$, where

$\widetilde{\Lambda}_{z}^{b}=\left(R_{\Sigma}^{*} \oplus R_{\Sigma}^{*}\right)\left(M_{z}^{b}\right)^{-1}\left(R_{\Sigma} \oplus R_{\Sigma}\right) \in \mathscr{B}\left(L^{2}(\Gamma) \oplus H^{-1 / 2}(\Gamma), L^{2}(\Gamma) \oplus H^{1 / 2}(\Gamma)\right), \quad z \in \mathbb{C} \backslash \mathbb{R}$. 
$\Delta_{\widetilde{\Lambda}^{b}}$ is a self-adjoint representation of the (bounded from above, this follows proceeding as in [24, page 1480]) Laplacian on $\mathbb{R}^{3} \backslash \bar{\Sigma}$ with boundary conditions at $\Sigma$ given by

$$
\left\{\begin{array}{l}
R_{\Sigma} \gamma_{0} u=b_{11}^{\Sigma} R_{\Sigma}\left[\gamma_{0}\right] u+b_{12}^{\Sigma} R_{\Sigma}\left[\gamma_{1}\right] u \\
R_{\Sigma} \gamma_{1} u=\left(b_{12}^{\Sigma}\right)^{*} R_{\Sigma}\left[\gamma_{0}\right] u+b_{22}^{\Sigma} R_{\Sigma}\left[\gamma_{1}\right] u
\end{array}\right.
$$

By [9, Theorem 2.19], the map $z \mapsto \widetilde{\Lambda}_{z}^{b}$ and the resolvent formula (3.5) extend to $Z_{\widetilde{\Lambda}^{b}}:=$ $\rho\left(\Delta_{\widetilde{\Lambda}^{b}}\right) \cap \mathbb{C} \backslash(-\infty, 0]$. Since $\sigma_{\mathrm{p}}^{-}\left(\Delta_{\widetilde{\Lambda}^{b}}\right)$ is empty (see [24, Theorem 3.7]),

$$
\left[\begin{array}{cl}
b_{11}^{\Sigma}+R_{\Sigma} \gamma_{0} S L_{\lambda}^{+} R_{\Sigma}^{*} & b_{12}^{\Sigma}+R_{\Sigma} \gamma_{0} D L_{\lambda}^{+} R_{\Sigma}^{*} \\
\left(b_{12}^{\Sigma}\right)^{*}+R_{\Sigma} \gamma_{1} S L_{\lambda}^{+} R_{\Sigma}^{*} & b_{22}^{\Sigma}+R_{\Sigma} \gamma_{1} D L_{\lambda}^{+} R_{\Sigma}^{*}
\end{array}\right]^{-1} \in \mathscr{B}\left(L^{2}(\Sigma) \oplus H^{-1 / 2}(\Sigma), L_{\bar{\Sigma}}^{2}(\Gamma) \oplus H_{\bar{\Sigma}}^{1 / 2}(\Gamma)\right)
$$

exists for any $\lambda \in(-\infty, 0)$ by Theorem [3.6. Therefore Theorem 5.6 applies to $F_{\lambda}^{\widetilde{\Lambda}^{b}}, \lambda \in$ $(-\infty, 0)$.

\section{REFERENCES}

[1] M. S. Agranovich: Strongly elliptic second order systems with spectral parameter in transmission conditions on a nonclosed surface. In: P. Boggiatto, L. Rodino, J. Toft, M. W. Wang (eds.), Pseudo-differential operators and related topics, Operator Theory: Advances and Applications, vol. 164, 1-21, Birkhäuser, Basel, 2006.

[2] M. S. Agranovich: Sobolev Spaces, Their Generalizations and Elliptic Problems in Smooth and Lipschitz Domains. Springer, Berlin, 2015.

[3] H. Baumgärtel, M. Wollenberg: Mathematical Scattering Theory, Akademie-Verlag, Berlin, 1983.

[4] J. Behrndt, M. Langer, V. Lotoreichik: Schrödinger operators with $\delta$ - and $\delta^{\prime}$-potentials supported on hypersurfaces. Ann. Henri Poincaré 14 (2013), 385-423.

[5] J. Behrndt, M. Malamud, H. Neidhardt: Scattering matrices and Dirichlet-to-Neumann maps. J. Funct. Anal. 273 (2017), 1970-2025.

[6] O. Bondarenko, X. Liu: The factorization method for inverse obstacle scattering with conductive boundary condition. Inverse Problems 29 (2013), 095021 (25pp).

[7] Y. Boukari, H. Haddar: The factorization method applied to cracks with impedance boundary conditions. Inverse Probl. Imaging 7 (2013), 1123-1138.

[8] J.F. Brasche, P. Exner, Y.A. Kuperin, P. Šeba: Schrödinger operators with singular interactions. J. Math. Anal. Appl. 184 (1994), 112-139.

[9] C. Cacciapuoti, D. Fermi, A. Posilicano: On inverses of Kreŭn's Q-functions, Rend. Mat. Appl. 39 (2018), 229-240.

[10] M. Costabel: Boundary Integral Operators on Lipschitz Domains: Elementary Results. SIAM J. Math. Anal. 19 (1988), 613-626.

[11] N. Grinberg: The Operator Factorization Method in Inverse Obstacle Scattering. Integr. equ. oper. theory 54 (2006), 333-348.

[12] G. C. Hsiao, W. L. Wendland: Boundary Integral Equations. Springer, Berlin, 2008.

[13] A. Jonsson, H. Wallin: Function Spaces on Subsets of $\mathbb{R}^{n}$. Math. Reports, 2 (1984), 1-221.

[14] K. Jörgens: Linear Integral Operators. Pitman, London, 1982.

[15] T. Kato: Scattering theory with two Hilbert spaces. J. Funct. Anal. 1 (1967), 342-369.

[16] T. Kato: Perturbation Theory for Linear Operators. Springer, Berlin, 1976

[17] A. Kirsch: Characterization of the shape of a scattering obstacle using the spectral data of the far field operator. Inverse Problems 14 (1998), 1489-1512.

[18] A. Kirsch, N. Grinberg: The Factorization Method for Inverse Problems. Oxford University Press, Oxford, 2008.

[19] A. Kirsch, A. Kleefeld: The factorization method for a conductive boundary condition. J. Integral Equations Appl. 24 (2012), 575-601. 
[20] A. Kirsch, S. Ritter: A linear sampling method for inverse scattering from an open arc. Inverse Problems 16 (2000), 89-105.

[21] A. Komech, E. Kopylova: Dispersion decay and scattering theory. John Wiley \& Sons, Hoboken, New Jersey, 2012.

[22] A. Mantile, A. Posilicano: Asymptotic Completeness and S-Matrix for Singular Perturbations, to appear in J. Math. Pures Appl. DOI: https://doi.org/10.1016/j.matpur 2019.01.017, 2019.

[23] A. Mantile, A. Posilicano, M. Sini: Self-adjoint elliptic operators with boundary conditions on not closed surfaces. J. Differential Equations 261 (2016), 1-55.

[24] A. Mantile, A. Posilicano, M. Sini: Limiting Absorption Principle, Generalized Eigenfunctions and Scattering Matrix for Laplace Operators with Boundary conditions on Hypersurfaces. J. Spectr. Theory 8 (2018), 1443-1486.

[25] A. Mantile, A. Posilicano, M. Sini: Uniqueness in inverse acoustic scattering with unbounded gradient across Lipschitz surfaces. J. Differential Equations 265 (2018), 4101-4132.

[26] W. McLean: Strongly elliptic systems and boundary integral equations. Cambridge University Press, Cambrdige, 2000.

[27] A. Posilicano: A Krĕn-like formula for singular perturbations of self-adjoint operators and applications. J. Funct. Anal. 183 (2001), 109-147.

[28] A. Posilicano: Boundary triples and Weyl functions for singular perturbations of self-adjoint operators. Methods Funct. Anal. Topology, 10 (2004), 57-63.

[29] A. Posilicano. Self-adjoint extensions of restrictions, Oper. Matrices, 2 (2008), 483-506.

[30] M. Schechter: Operator Methods in Quantum Mechanics. North Holland, New York, 1981 (Dover reprint, 2002).

[31] M. Reed, B. Simon: Methods of Modern Mathematical Physics. Vol. III Scattering Theory. Academic Press. London 1979.

[32] E. P. Stephan: Boundary integral equations for screen problems in $\mathbb{R}^{3}$. Integral Equations Operator Theory. 10 (1987), 236-257.

[33] H. Triebel: Fractals and Spectra. Birkhäuser. Basel, 1997.

[34] D. R. Yafaev. Mathematical Scattering Theory. General theory. American Mathematical Society, 1992.

[35] C. H. Wilcox: Scattering theory for the d'Alembert equation in exterior domains. Lecture Notes in Mathematics, Vol. 442. Springer, Berlin 1975.

Laboratoire de Mathématiques, Université de Reims - FR3399 CNRS, Moulin de la Housse BP 1039, 51687 Reims, France

DiSAT, Sezione di Matematica, Università dell'Insubria, via Valleggio 11, I-22100 Como, ITALY

E-mail address: andrea.mantile@univ-reims.fr

E-mail address: andrea.posilicano@uninsubria.it 